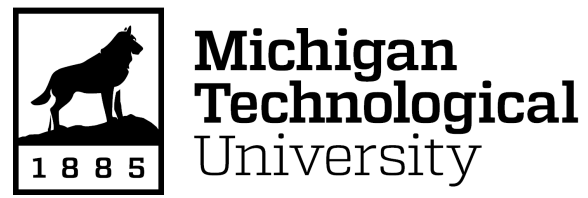

Michigan Technological University Digital Commons @ Michigan Tech

Dissertations, Master's Theses and Master's Reports

2021

\title{
Application of Carbon Nanotube Thermophones as Duct Noise Cancelling Speakers: Using New Technology with Old Theories
}

Stephania M. Vaglica

Michigan Technological University, smvaglic@mtu.edu

Copyright 2021 Stephania M. Vaglica

\section{Recommended Citation}

Vaglica, Stephania M., "Application of Carbon Nanotube Thermophones as Duct Noise Cancelling Speakers: Using New Technology with Old Theories", Open Access Master's Report, Michigan Technological University, 2021.

https://doi.org/10.37099/mtu.dc.etdr/1224

Follow this and additional works at: https://digitalcommons.mtu.edu/etdr

Part of the Acoustics, Dynamics, and Controls Commons 


\title{
Application of Carbon Nanotube Thermophones as Duct Noise Cancelling Speakers: Using New Technology with Old Theories
}

\author{
By \\ Stephania Vaglica \\ A REPORT \\ Submitted in partial fulfillment of the requirements for the degree of \\ MASTER OF SCIENCE \\ In Mechanical Engineering \\ MICHIGAN TECHNOLOGICAL UNIVERSITY \\ 2021 \\ (C) 2021 Stephania M. Vaglica
}


This report has been approved in partial fulfillment of the requirements for the Degree of MASTER OF SCIENCE in Mechanical Engineering.

Department of Mechanical Engineering - Engineering Mechanics

Thesis Advisor: Dr. Andrew Barnard

Committee Member: Dr. James DeClerck

Committee Member: Dr. Jason Blough

Department Chair: William W. Predebon 


\section{Table of Contents}

Application of Carbon Nanotube Thermophones as Duct Noise Cancelling Speakers:

Using New Technology with Old Theories

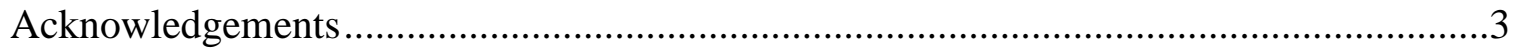

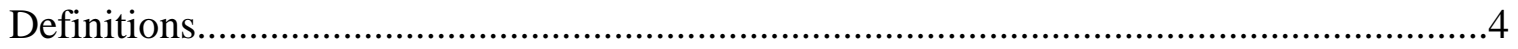

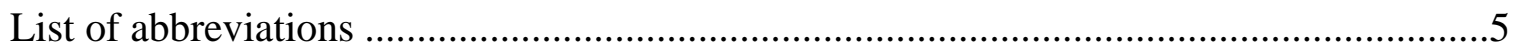

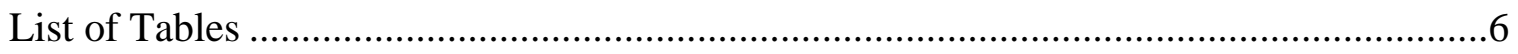

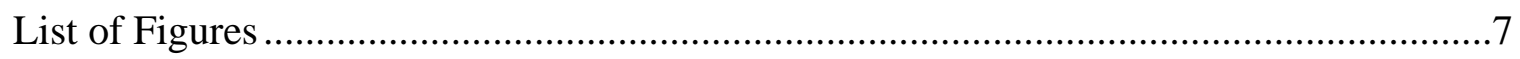

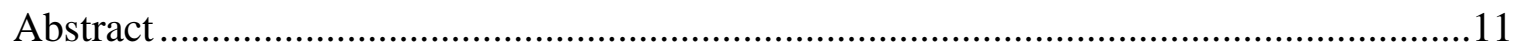

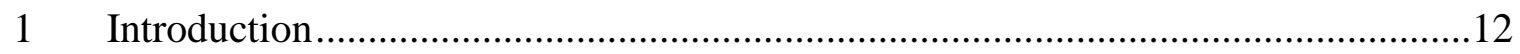

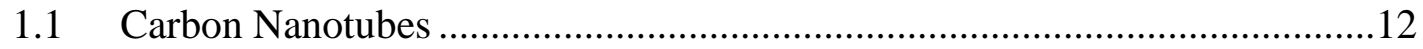

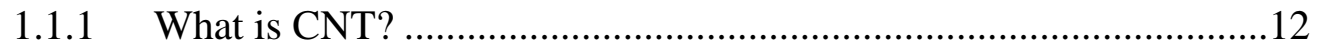

1.1.2 CNT as a Thermophone ...........................................................12

1.1.2.1 CNT Thermophones..................................................12

1.1.2.2 CNT Heat Management ..............................................13

1.1.2.3 CNT Efficiency .....................................................14

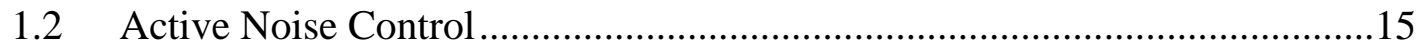

1.2.1 What is Active Noise Control? ....................................................15

1.2.1.1 Pipe and Duct Noise Control ......................................16

1.2.1.2 CNT as an Active Noise Controller ..............................17

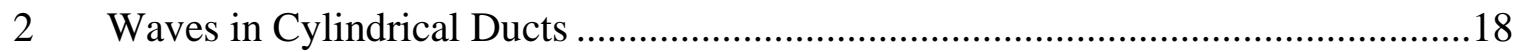

2.1 General Propagations in Circular Ducts .....................................................18

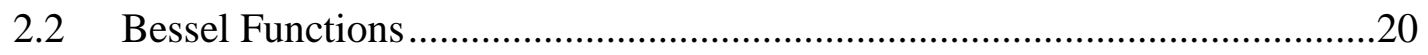

2.3 How Non-Plane Waves Are Generated......................................................23

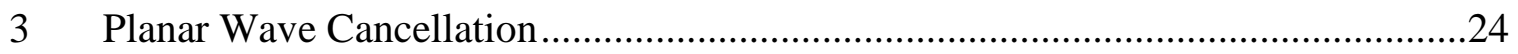

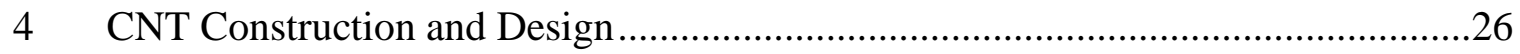

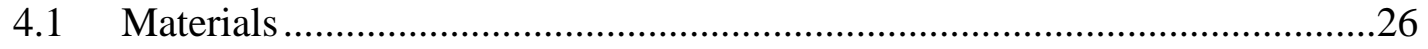

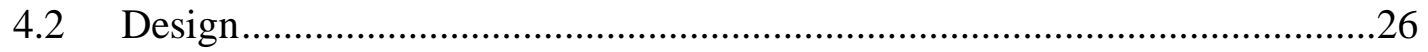

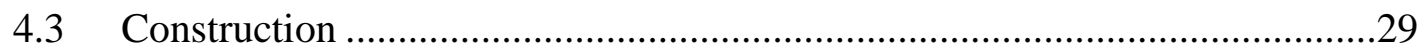


5 Methods and Materials .32

$5.1 \quad$ Planar Wave Cancellation Setup …………....................................................32

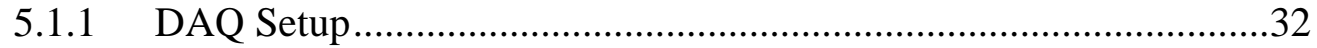

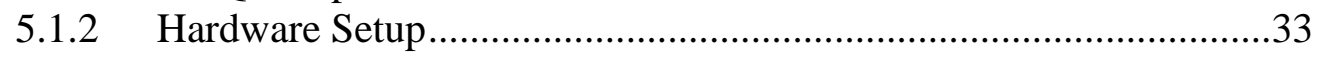

5.1.3 ANC Setup .......................................................................34

5.1.4 Extended Planar Wave Cancellation..............................................36

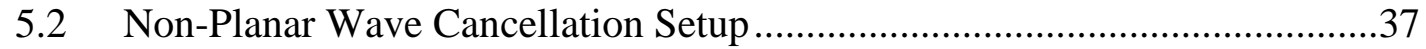

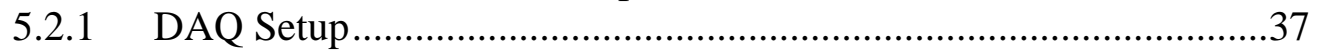

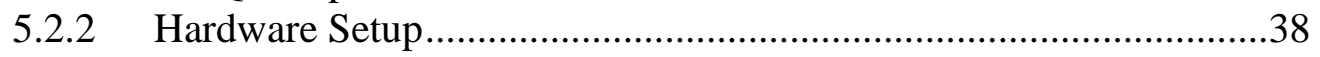

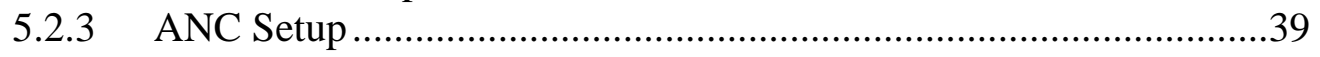

$5.3 \quad$ Accelerated Life Testing Setup ......................................................... 40

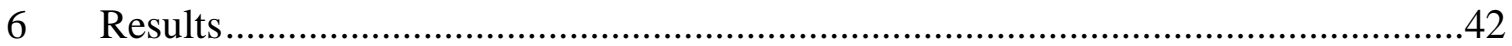

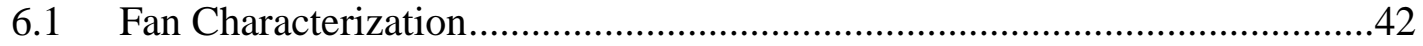

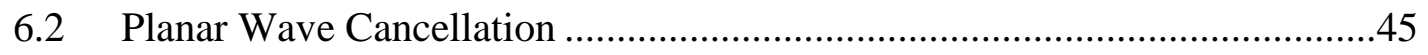

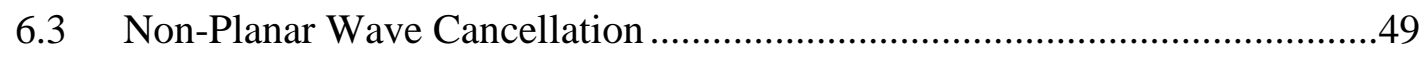

6.3.1 Centered in the pipe ...........................................................49

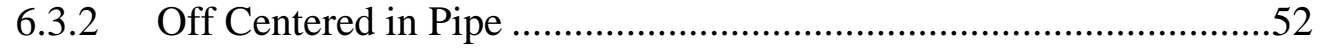

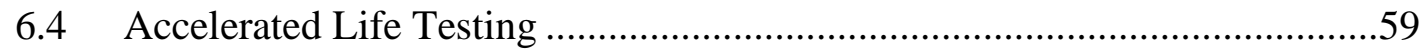

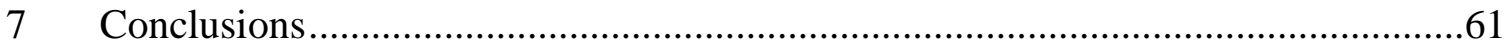

7.1.1 Speaker Construction ............................................................61

7.1.2 ANC Planar Cancellation.........................................................61

7.1.3 ANC Non-planar cancellation...................................................62

7.1.4 Accelerated Life Testing ..........................................................62

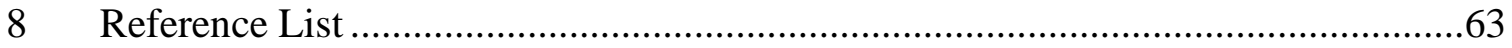




\section{Acknowledgements}

I would like to say that between typical stress of grad school and the Covid-19 pandemic, there are a multitude of people I would like to thank. I would like to start off by thanking my advisor and mentor, Dr. Andrew Barnard. He has done more for my education than any person I can think of. Andrew has been a constant support for any of my crazy ideas and endeavors and has learned to never order a Tidal Wave again. I would like to thank Steven Senczyszyn for his aid in helping me build the CNT speakers that were used for this project and his guidance on designing. I would like to thank Walker Nelson for sharing his design ideas to help the project move along quickly and for also being one of my closest grad school friends. I would like to thank Kevin Nelson and GLSV for providing the controller technology and for helping me test until the early hours of the morning.

I would like to thank my committee members, Jim DeClerck and Jason Blough, for dedicating their time to be on my committee, and for their guidance in class. Both professors have taught me so much in such a small amount of time, especially professor DeClerck. His open-door policy that I abused to eat candy ended up being very fun and informative.

I would like to thank my lab group for being a constant source of fun and support and making my time at school so enjoyable.

And most importantly, I would like to thank my family, Stephen Vaglica and Mari Freundl. I would like to thank them for all the 8-hour trips they made to visit, and for being there for me during my grad school time. My lovely grandparents, my siblings PJ and Catie, and my friends, especially Kerri Gudenau, have been a constant support as well. I can say with certainty, that it takes a village to raise a child.

Thank you. 


\section{Definitions}

Sound pressure level: Measurement of what humans hear. This measurement is typically represented in pascals or decibels.

Order: A number of times a given event is occurring. It is found by transforming the time domain signal to the angular domain.

ANC (Active Noise Control): The ability to reduce the noise level in a room by playing the same signal $180^{\circ}$ out of phase.

Thermoacoustic Effect: The ability for specific materials to produce sound using heat.

Planar waves: Waves that propagate as a plane down a waveguide.

Non-planar waves: Waves that have a multi-phase component and geometric shape, that propagate down a waveguide.

Cut-on frequency: The frequency at which planar waves transition into non-planar waves. 


\section{List of abbreviations}

CNT Carbon Nanotubes

ANC Active Noise Control

FXLMS Filtered-X Least Mean Squares

HVAC Heating, Ventilation, and Air Conditioning

FRF Frequency Response Function

GLSV Great Lakes Sound and Vibration

HCPUA Heat Capacity Per Unit Area 


\section{List of Tables}

Table 1. The bill of materials for creating the speaker .............................................29

Table 2. LMS Data Acquisition Hardware Parameters .................................................32

Table 3. Planar Wave Testing Data Acquisition Sampling Parameters............................32

Table 4. LMS Data Acquisition Hardware Parameters ................................................37

Table 5. Planar Wave Testing Data Acquisition Sampling Parameters.............................37

Table 6. LMS Data Acquisition Hardware Parameters ................................................40

Table 7. Accelerated Life Testing Acquisition Sampling Parameters. ............................40 


\section{List of Figures}

Fig. 1 CNT forests grown using chemical vapor deposition. A) shows the CNT on a chip that is roughly 250 micrometers tall. B) and C) show various magnifications of A taken with a SEM microscope [1] (reproduced with permission from professor Aliev)

Fig. 2 A planar CNT speaker. The black nanofilm is wrapped around two copper electrodes which creates the body of the speaker [8].

Fig. 3 Surface temperature of flat panel CNT speaker at $5 \mathrm{~cm}$ away for a $110 \mathrm{~W} 2000 \mathrm{~Hz}$ signal [14].

Fig. 4 Feedforward FXLMS algorithm for an ANC system using any speaker or microphone combination [21].....

Fig. 5 Traditional ANC system with incorporated branch pipe design and loudspeaker [7].

Fig. 6 Bessel functions generated in MATLAB

Fig. 7 Circular duct mode shapes generated from Bessel functions. $M$ is the number of circumferential modes and $\mathrm{N}$ is the number of radial modes. This matrix shows the pressure fluctuations of the gray and white regions [28].

Fig. 8 A plane wave is sent through a hard wall duct from the point source on the left. The pipe duct is small enough and the frequency is low enough that only plane waves can propagate [28].

Fig. 9 An off-axis point source (left dot) is now allowing the $(0,1)$ mode to propagate in the pipe. The pressure is no longer planar over time [28].

Fig. 10 The frequency and duct diameter have been changed which allows the $(1,0)$ mode to form. The "donut" mode is the alternating pressures down the duct caused by parameter changes, even though the point source is centered [28]....

Fig. 11 Downstream cancellation visualization. The fan disturbance (blue) and the cancellation signal (orange) will meet and cause a destructive interference sum (green) between the two signals.

Fig. 12 The wave that travels toward the noise disturbance. It creates a standing wave which adds when the cancellation signal and disturbance signal meet.

Fig. 13 Copper tape electrode configuration.

Fig. 14 Electrode pattern created using copper tape and adhered to PTFE FG. 
Fig. 15 A quick guide to constructing a CNT speaker.

Fig. 16 CNT speaker taped into the duct system. The speaker is not taped down all the way to better show its flexibility in the speaker.

Fig. 17 A side profile of duct setup with their lengths

Fig. 18 The planar wave cancellation setup with the 8-blade fan installed in the rear. The GLSV measurement system is connected to the error microphone and sends a drive signal to the CNT speaker to cancel noise from the fan.

Fig. 19 Lab setup for planar wave cancellation testing. .34

Fig. 20 The user interface for the GLSV ANC controller. .35

Fig. 21 The non-planar wave cancellation. An addition of a frequency generator, amplifier, and loudspeaker in lieu of a fan.

Fig. 22 The inline test setup (right) and non-inline test setup (left).

Fig. 23 A picture of the accelerated life test setup

Fig. 24 Order tracking sound data of the 8-bladed fan

Fig. 25 Frequency spectra of the low (red), medium (green), and high (blue) settings on the fan.

Fig. 26 Order tracking done on the fan with a microphone situated 1 meter away at a $45^{\circ}$ angle away from the flow path.

Fig. 27 Coherence of the ANC system prior to testing.

Fig. 28 Sound cancellation of $8^{\text {th }}$ order $(314 \mathrm{~Hz})$ tone. Red data shows cancellation off and green shows cancellation on. The $8^{\text {th }}$ order tone is eliminated.

Fig. 29 An A-weighted 1/3 octave representation of the $8^{\text {th }}$ order ton cancellation. Here the green data is the cancellation off and blue data is the cancellation on. It is important to note that the $360 \mathrm{~Hz}$ tone was filtered out in this data.

Fig. 30 A $24^{\text {th }}$ order cancellation test. Red data is cancellation off, and green data is cancellation on. The frequency of interest was $942 \mathrm{~Hz}$.

Fig. $318^{\text {th }}(314 \mathrm{~Hz})$ and $16^{\text {th }}(627 \mathrm{~Hz})$ orders cancellation data. Here the red shows the cancellation off and the cyan shows the cancellation on.

Fig. 32 A three-minute stability test for the cancellation of $8^{\text {th }}$ and $16^{\text {th }}$ orders. The red shows the cancellation off and the green shows the cancellation on. 
Fig. 33 Cancellation of a $1250 \mathrm{~Hz}$ tone that is before the cutoff frequency. The red and green data are cancellation off and the blue and magenta are two cancellation on runs.

Fig. 34 Cut on Frequency cancellation test. Here, the red and green show cancellation is off, and the magenta and cyan show cancellation is on at the $1318 \mathrm{~Hz}$ tone.........50

Fig. 35 Cancellation above the cut-on frequency at $1400 \mathrm{~Hz}$. The red data shows cancellation off and blue data shows cancellation on.

Fig. 36 Cancellation at $1500 \mathrm{~Hz}$. The red shows cancelation off, and the green data shows cancellation on.

Fig. 37 Off centered cancellation of the $1200 \mathrm{~Hz}$ tone. Red and green are the cancellation off runs and the blue and magenta are cancellation on runs.

Fig. 38 1/3 Octave map of the two cancellation tests. The blue and green bars show cancellation off and the cyan and brown bars show cancellation on.

Fig. 39 Cancellation at $1318 \mathrm{~Hz}$ with disturbance off centered. Red and green show the cancellation off and blue and magenta show cancellation on.

Fig. 40 1/3 Octave band spectra of cancellation at $1318 \mathrm{~Hz}$ with disturbance off centered. Blue and green show the cancellation off and cyan and magenta show cancellation on

Fig. 41 Cancellation at $1400 \mathrm{~Hz}$ with disturbance off centered. Red shows the cancellation off and green shows cancellation on......

Fig. 42 1/3 Octave band spectra of cancellation at $1400 \mathrm{~Hz}$ with disturbance off centered. Green shows the cancellation off and blue shows cancellation on. .56

Fig. 43 Downstream testing of $1318 \mathrm{~Hz}$ tone. The red indicated the near duct microphone measurement with cancellation off. The green shows the near duct microphone with cancellation on. Blue shows the downstream microphone with cancellation off and magenta shows the downstream microphone with cancellation on.....

Fig. 44 Downstream testing of $1400 \mathrm{~Hz}$ tone. The red indicated the near duct microphone measurement with cancellation off. The green shows the near duct microphone with cancellation on. Blue shows the downstream microphone with cancellation off and magenta shows the downstream microphone with cancellation on......

Fig. 45 Downstream testing of $1500 \mathrm{~Hz}$ tone. The red indicated the near duct microphone measurement with cancellation off. The green shows the near duct microphone with cancellation on. Blue shows the downstream microphone with cancellation off and magenta shows the downstream microphone with cancellation on 
Fig. 46 Sound pressure at $20 \mathrm{KHz}$ recorded at 10 minute increments (left) and voltage and current measurements taken at 1 minute increments (right)........................59

Fig. 47 Various images of a failed CNT speaker that burned out due to lack of cooling..60 


\section{Abstract}

Certain materials can produce sound through heat fluctuations. In recent times, the material capable of this was discovered to be Carbon Nanotubes (CNT). The idea is to create a flexible speaker that can be made into any shape or form to create sound. Once a speaker has been created, an active noise control system can be designed to cancel loud sounds in building and pipe ducts. This technology, similar to noise cancelling headphones, allows a user to create a modular system to mitigate noise in everyday life. Thus, the purpose of this research is to illustrate the abilities of a CNT speaker system to perform active noise control on an HVAC duct application. This paper will start with a brief introduction into CNT technology and thermophone acoustics before diverging into active noise control technology. It will illustrate planar and non - planar wave cancellation and showcase a small scale accelerated life test. 


\section{Introduction}

\subsection{Carbon Nanotubes}

\subsubsection{What is CNT?}

Carbon Nanotubes are hexagonal allotropes of carbon that are 1/1000 the thickness of a human hair [1]. They are created through various means, although most commonly created through a process called chemical vapor deposition [2]. During this process, high temperature gasses are put into a pressure chamber and heated until the carbon "falls" out of the air and grows on a special silicone plate [1].The tubes begin to grow taller the longer that the chemical process continues. This forms what's called a "Carbon Nanotube Forest" [3]. Figure 1 illustrates the CNT forests. CNT forests are called so because when nanozooming onto the silicon chip, one can see what looks like multi-walled nanotubes standing tall like trees [3]. These "trees" will fall and pull on other trees, which creates a smooth nanofilm that can be used for multiple purposes [1]. The purpose is the ability for CNT to act as a thermophone speaker.
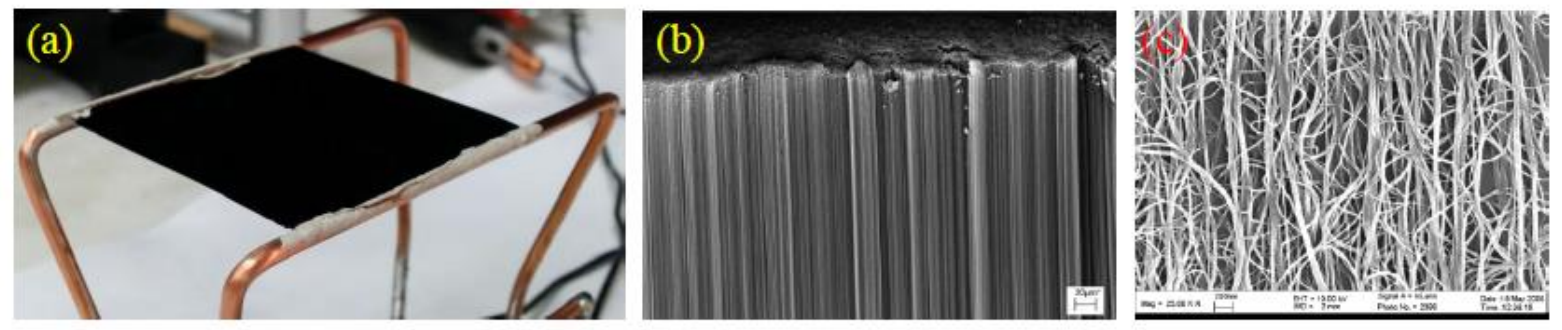

Fig. 1 CNT forests grown using chemical vapor deposition. A) shows the CNT on a chip that is roughly 250 micrometers tall. B) and C) show various magnifications of A taken with a SEM microscope [1] (reproduced with permission from professor Aliev).

CNT also has the unique property of being highly flexible [4]. Since the material is nano and has the highest tensile strength of any material [5] [6], CNT films are able to conform to any shape or size making it an ideal material for odd shapes such as cylinders and spheres that traditional loudspeakers are unable to make. They can also be made to accommodate nanoscale speakers or speakers as large as a wall in a house, depending on application [7].

\subsubsection{CNT as a Thermophone}

\subsubsection{CNT Thermophones}

One of the properties that make CNTs unique is the ability to produce sound via thermoacoustic effect. The thermoacoustic effect is a property of a material in which it can heat and cool rapidly [8] [5] [9]. This quick succession of heating and cooling will allow 
the air to expand and contract, which creates pressure variations [8]. These variations form a pressure wave that humans can hear. The effect was first theorized in 1898 by Braun who detailed how heat can produce sound [10]. The effect was further studied by scientists Arnold and Crandall who explored this with platinum [8] [10]. Unfortunately, platinum cannot heat and cool fast enough to create frequencies that humans can hear or, that are useful in applications of sound. For nearly 100 years, the research on thermophones was effectively dead until 2008, when a research team in China accidentally discovered sound through CNT [8] [11] [12]. CNT has an ultra-low heat capacity per unit area (HCPUA) that allows it to change temperatures instantly [13]. CNT speakers can create sound up to 100 $\mathrm{kHz}$, which makes it usable for ultrasonic applications as well [8]. With this new nanomaterial and newly discovered phenomena, the ability to create a CNT thermophone became achievable and research in this field increased. Although still a new field, the last decade has seen a large expansion of experiments and papers on CNT thermophones. Figure 2 shows a typical "flat" CNT speaker.
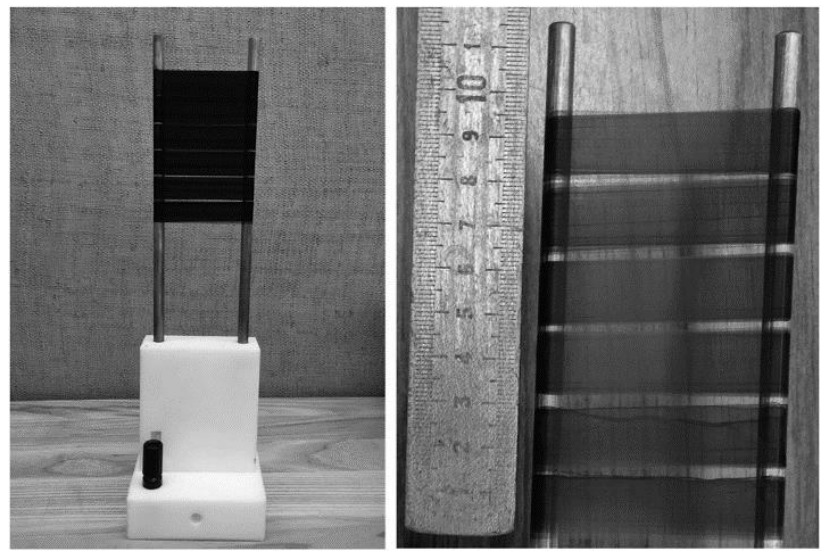

Fig. 2 A planar CNT speaker. The black nanofilm is wrapped around two copper electrodes which creates the body of the speaker [8].

\subsubsection{CNT Heat Management}

It may seem apparent that since CNT speakers use heat to produce sound, there is an inherent issue with heat management to reduce the risk of fire hazards. One researcher, Mahsa Asgarisabet, found that measuring at $5 \mathrm{~cm}$ away, the speaker can be as hot as $65^{\circ} \mathrm{C}$ with 110 Watts of electrical input power [14]. Using nearfield acoustic holography, a process that uses a microphone array mounted near the surface to measure the vibration of the source in 3D space, the researcher was able to accurately ascertain the surface temperature of the CNT speakers, which had previously been unattainable due to their rapid temperature fluctuations. They demonstrated how temperature will increase as a function of power, which means that the louder the speaker is, the hotter the surface of the speaker will get [14]. Their results are illustrated in figure 3 . 


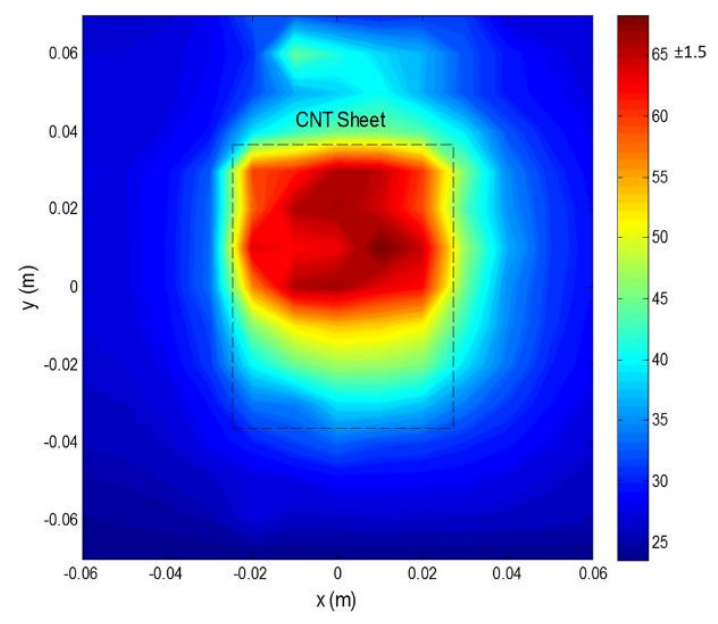

Fig. 3 Surface temperature of flat panel CNT speaker at $5 \mathrm{~cm}$ away for a $110 \mathrm{~W} 2000 \mathrm{~Hz}$ signal [14].

Lead CNT researcher, Aliev, has looked at ways of managing the heat from the speakers [15]. Carbon also has the unique ability of being able to withstand exceedingly high temperatures. To prevent burning, Aliev proposes that CNT can be layered between two resonant plates with inert gas inside, which will prevent combustion with oxygen [15]. This also increased the sound output because both the plates resonant at the frequency that the CNT speaker outputs which results in a higher sound pressure value. This paper provides valuable design information on how to produce a CNT speaker that will not combust or burn materials around it while increasing the volume of the speaker.

\subsubsection{CNT Efficiency}

Now that it has been established that CNT speakers are possible, the practical efficiency of the speakers needs to be addressed. One source experimentally quantified the efficiency of a CNT speaker finding that the efficiency ranged from $4.3 \times 10^{\wedge}-6$ to $319 \times$ $10^{\wedge}-6 \%$ efficient [8]. This efficiency is quite low, being significantly less than $1 \%$ efficient. Even compared to a typical loudspeaker, which ranges from 1-4\% efficiency [16], CNT speakers are not efficient producers of sound, which means that more power is needed to drive them [17]. This can lead to design issues where the voltage needed to use the speaker exceeds $120 \mathrm{~V}$, which is outside the voltage range of houses in the U.S. Aliev also proposes how encapsulating CNT film between two sheets with inert gas will improve the efficiency [16]. During his research he found he could improve the efficiency by $0.1 \%$ in air with his encapsulation model [16]. The encapsulation model is a speaker that is enclosed in a membrane of a given material. Despite the small increase, this is a fairly substantial gain in efficiency considering this would result in a $10 \mathrm{~W}$ increase in efficiency on a power driver signal of $1 \mathrm{kWatt}$. Aliev was able to achieve this using his thermal management technique of creating free hanging nanotubes inside two resonant plates with inert gas for combustion prevention and temperature gradient increase [16]. This shows demonstrates 
these speakers can be used for sound production in active noise control applications. The last thing to consider is thermal efficiency of CNT speakers. Recent research suggests that by utilizing the nanosized properties of CNT, the heat efficiency can be increased [18]. Since sound pressure is inversely proportional to the heat capacity of CNT, the heat capacity needs to be maximized to allow for greater sound generation [18]. Furthermore, the heat efficiency at high frequencies appears to be limited to the heat capacity of the electrode wires used to create the speaker [18]. These are further effects that need to be accounted for when optimized the heat management of CNT thermophones.

\subsection{Active Noise Control}

\subsubsection{What is Active Noise Control?}

Active noise control is the ability to cancel sound through destructive interference [19] [20]. The principle is simple: an undesired noise wave occurs at some point in space where it is measured. Then an "anti-noise" signal is played that meets the original sound wave and cancels it [21]. The anti-noise wave is the same signal as the original noise; however, it is 180 degrees out of phase. The superposition of the two waves results in a greatly reduced noise level. Researchers Hu and Rajamani explored various ways to create this active noise control (ANC) system [21]. One of the most effective ways was to use a feedforward filtered X least mean squared projection (Feedforward FXLMS) [22]. This is effective for its ease of implementation and its ability to apply an error correction constantly throughout the algorithm, which ensures proper phase alignment of the two superimposed waves [21]. Figure 4 shows the flowchart for the feedforward FXLMS algorithm. The reference, or noise signal is fed into a function that finds the transfer path between the noise signal and the measurement transducer $(\mathrm{P}(\mathrm{z}))$. The LMS algorithm finds the coefficients needed to filter the noise signal to make it out of phase [20]. This is fed into the $\mathrm{W}(\mathrm{z})$ filter function where the wave is filtered and becomes anti-noise [21]. The anti-noise signal then has its own transfer path $(\mathrm{S}(\mathrm{z}))$ to the transducer since this wave has to be generated by the speaker [21]. At the residual noise junction, the waves are added and canceled, and any residual noise is fed into the algorithm to constantly improve and correct for noise [21]. This process repeats indefinitely while the active noise control system is turned on. 


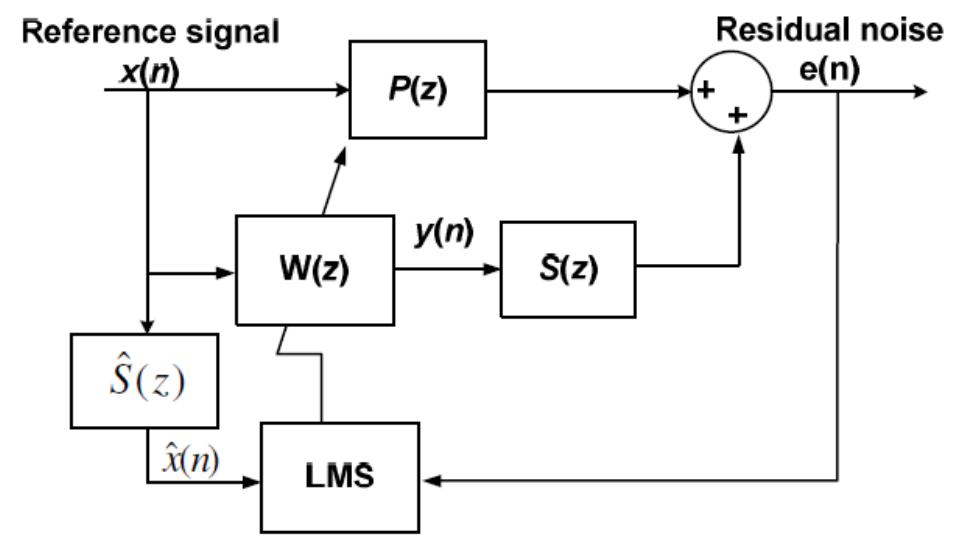

Fig. 4 Feedforward FXLMS algorithm for an ANC system using any speaker or microphone combination [21].

This is the most promising as it requires few pieces of equipment and provides high accuracy; therefore, this ANC model is the one that will be implemented into the CNT speaker design.

\subsubsection{Pipe and Duct Noise Control}

In a pipe, the shape of the acoustic field changes to reflect circular modes. These modes propagate through the duct as planar and non-planar modes [23]. This means, to cancel noise in a duct, one must cancel the modes at given frequencies. Modes in a duct can be found by using the respective eigenfunctions with the cross-sectional area of the duct to find the mode and mode cutoff frequency [23] [19]. This cutoff frequency is imperative to find before testing because any noise that is created above this cutoff frequency will be difficult to cancel and is not guaranteed to reduce the sound [23]. The cutoff frequency is a calculated quantity and is a function of the geometry of the duct. Although feedforward algorithm is the most commonly used, one researcher, Jean Claude Carmona, looked at applying feedback techniques in a duct instead of feedforward techniques to increase the long-term robustness of the algorithm on the noise signal [24]. This is due to the compact pressure variations in a duct where the air is confined to a specific shape and typically propagates in one direction like a standing wave. This researcher, instead, applied state space models to linearly predict the 1D state-space system [24]. Despite his claims for robustness, this method would require a high understanding of the duct before the system is implemented, which would make it exceedingly difficult to sell a "one size fits all" commercial solution [24]. It would also require complex modeling of each system and the need to choose specific parameters and boundary conditions. Jean Claude Carmona describes some interesting alternatives if the FXLMS algorithm does not work. Armed with this information, the algorithm can be created specifically with duct models in mind, but using a CNT speaker instead of a traditional loudspeaker. 


\subsubsection{CNT as an Active Noise Controller}

The culmination of all the information discussed leads to the idea that CNT speakers have the potential to be active noise controllers in pipe ducts. Aliev proposes this exact marriage between ANC systems and CNT systems in one of his most recent papers [1]. The paper details work about sonar arrays, which is not the application that is discussed; however, it sheds light on the ability of CNT ANC systems [1]. CNT films are lightweight and have the ability to survive high temperature environments. This gives them advantages for noise cancellation in car exhaust pipes where the hot gasses from the car are expelled loudly. Similarly, the major advantage of a CNT speaker is that it does not need branching pipes for speakers to generate sound. The flexible CNT speaker can wrap around the pipe and generated circumferential pressure and mode cancellation. Figure 5 demonstrates what a traditional pipe ANC cancellation system looks like.

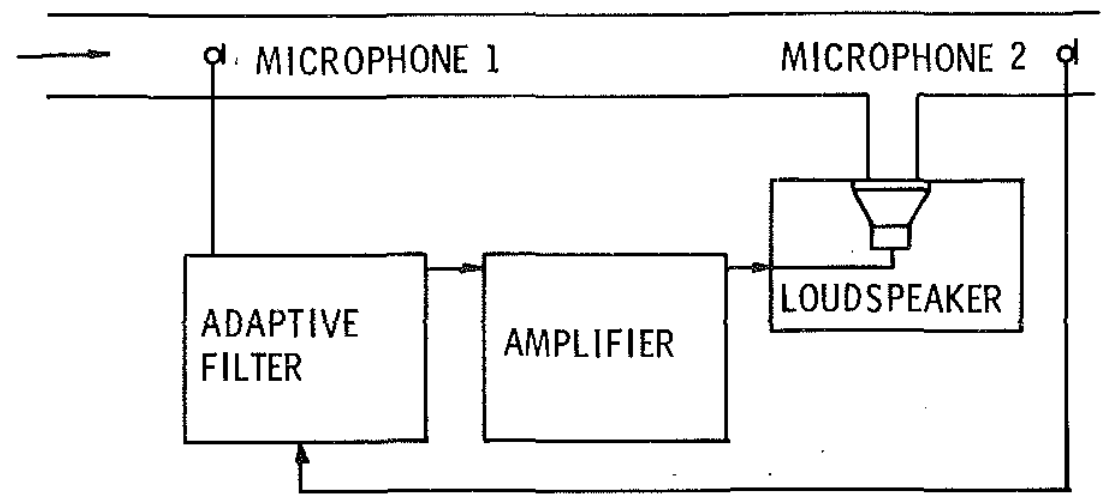

Fig. 5 Traditional ANC system with incorporated branch pipe design and loudspeaker [7].

The CNT speaker would not require the loudspeaker that branches and can make it difficult to install into pre-existing systems. The CNT speaker will, instead, be able to conform to any size by simply installing it in the duct. These advantages make it a strong candidate to replace conventional ANC systems in pipes and ducts and instead replace it with a CNT modular speaker.

The objective of this research is to take the principles of ANC systems and apply them to CNT speakers to mitigate noise issues in HVAC applications. Although CNT active noise cancellation has been proposed in buildings and solar applications [7], this researched is focused on finding the viability in HVAC applications, specifically tonal dominated HVAC fan noise. The first step is to create a CNT speaker that will be loud enough to cancel the signal, and then test the CNT speaker's ability to cancel with ANC. The test will attempt to cancel both planar waves, and non-planar waves. After testing, a long-term durability test will be performed on the speaker to see if this is a viable solution to noise problems in a duct. 


\section{Waves in Cylindrical Ducts}

\subsection{General Propagations in Circular Ducts}

Waves form when air particles travel down a duct in an oscillating motion. This motion is tied very specifically to the geometry of the duct, and the frequency of the waves generated. This section will illustrate how waves form in a duct as non-planar waves. Then, certain special cases, such as how planar waves can propagate in a duct, will be mathematically observed in the next section.

The general treatment below was derived using sources which specify wave propagation [25] [26]. Using polar coordinates for the ease of calculating a circular duct wave, the wave equation is written as

$$
\nabla^{2} p=\frac{1}{c^{2}}\left(\frac{\partial^{2} p}{\partial t^{2}}\right)
$$

where $p$ is the pressure field, $c$ is the speed of sound in air, and $t$ is the time. By assuming that the wave must follow an $e^{j \omega t}$ time dependence, this can be reduced to the Helmholtz equation which shows that

$$
\nabla^{2} p+k^{2} p=0
$$

where the pressure field of the wave is propagating down and is a function of the radius of the duct, $r$, the angle, $\theta$, and the longitudinal distance of the wave, $z: p(r, \theta, z)$. As shown, there is now no time dependence on $p$ now because of the time dependence assumption made. $k$ is the wave number which is the spatial frequency of a wave (cycles/distance). It is defined by

$$
k=\frac{2 \pi}{c}=\frac{\omega}{c}
$$

An expansion of equation (2) yields the Laplacian operator in polar coordinates where

$$
\frac{\partial^{2} p}{d r^{2}}+\frac{1}{r} \frac{\partial p}{\partial r}+\frac{1}{r^{2}} \frac{\partial^{2} p}{\partial \theta^{2}}+\frac{\partial^{2} p}{\partial z^{2}}+k^{2} p=0
$$

And by using separation of variables, one will get 


$$
p(r, \theta, z)=p_{r}(r) p_{\theta}(\theta) p_{z}(z)
$$

and applying the boundary conditions at rigid walls leaves

$$
\left(\frac{\partial p}{\partial r}\right)_{r=a}=0
$$

which states that at the outer radius of the duct, the fluctuating particle velocity field is zero. Then, substituting equation (5) back into equation (4), the formula then becomes

$$
p_{\theta} p_{z} \frac{\partial^{2} p_{r}}{d r^{2}}+p_{\theta} p_{z} \frac{1}{r} \frac{\partial p_{r}}{\partial r}+p_{r} p_{z} \frac{1}{r^{2}} \frac{\partial^{2} p_{\theta}}{\partial \theta^{2}}+p_{\theta} p_{r} \frac{\partial^{2} p_{z}}{\partial z^{2}}+k^{2} p_{\theta} p_{r} p_{z}=0
$$

To get the $k^{2}$ term alone, the equation (7) is divided by $p_{\theta} p_{r} p_{z}$. This also allows for the $r$ terms, $\theta$ terms, and $z$ terms to group together making it easier to solve for them individually. Further, the entire equation can be multiplied by $r^{2}$ to remove the $r$ term from the $\theta$ term leaving

$$
r^{2} \frac{1}{p_{r}} \frac{\partial^{2} p}{d r^{2}}+\frac{1}{p_{r}} r \frac{\partial p}{\partial r}+\frac{1}{p_{\theta}} \frac{\partial^{2} p}{\partial \theta^{2}}+\frac{1}{p_{z}} \frac{\partial^{2} p}{\partial z^{2}}+k^{2} r^{2}=0
$$

Since $k^{2} r^{2}$ must be a constant, it can be logically assumed that the $r$ terms and the $\theta$ terms are also constants that have to equal each other. By assigning a trivial constant to the equations, $\mathrm{m}^{2}$. This will create 3 equations (9) (10) (11), one with $r$ terms, one with $\theta$ terms, and one with $z$ terms:

$$
\begin{gathered}
\frac{1}{p_{\theta}} \frac{\partial^{2} p_{\theta}}{\partial \theta^{2}}=-m^{2} \text { or } \frac{1}{p_{\theta}} \frac{\partial^{2} p_{\theta}}{\partial \theta^{2}}+m^{2} p_{\theta}=0 \\
r^{2} \frac{1}{p_{r}}\left[\frac{\partial^{2} p_{r}}{d r^{2}}+\frac{1}{r} \frac{\partial p_{r}}{\partial r}\right]+k_{m n}^{2} r^{2}=m^{2} \\
\frac{\partial^{2} p_{z}}{\partial p_{z}^{2}}=-k_{z l}^{2} Z
\end{gathered}
$$

This becomes increasingly important later as equations (9) and (10) will contribute to the mode shape of the duct. 
Further, it is important to note that the total magnitude of the wave number $k$ is found by summation of the axial wave number, $k_{z l}$, where $z l$ is the total length boundary of the cylindrical duct, and $k_{m n}$, where $m n$ are the Bessel functions values. This would create a total $\mathrm{k}$ value of

$$
k^{2}=k_{m n}^{2}+k_{z l}^{2}
$$

Finally, the three equations (9), (10) and (11) have the solutions of

$$
\begin{gathered}
p_{z}=\cos \left(k_{z l} z\right) \\
p_{\theta}=\cos \left(m \theta+\gamma_{l m n}\right) \\
p_{r}=J_{m}\left(k_{m n} r\right)
\end{gathered}
$$

where $\gamma_{l m n}$ is the phase angle of the wave, and $J_{m}$ is a Bessel function and are described further detail in the next section.

\subsection{Bessel Functions}

The bracketed portion of equation (10) is better known as a Bessel equation. A Bessel function, otherwise known as a cylinder function, describes the solution to Laplace's equation using cylindrical coordinates [26]. They also describe solutions to Helmholtz's equation in spherical coordinates [26]. These Bessel functions become important because they describe the motion and shape of a cylindrical or circular duct. To solve these Bessel equations, there are two types of Bessel functions used: Bessel functions of the first kind, and Bessel functions of the second kind. A Bessel function (shown below) crosses the zero on $\mathrm{x}$-axis an infinite number of times, meaning it contains an infinite number of zeros. 




Fig. 6 Bessel functions generated in MATLAB

The total general solution of a Bessel equation includes the combination of the Bessel function of the first kind and the second kind; however, the coefficients of the second kind go to zero for a rigid duct because the boundary conditions at the edge of the duct are zero. This means that for a circular membrane with a rigid duct, the solutions are Bessel functions of the first kind. There are two equations for calculating Bessel functions of the first kind. The first is for when $m$ is equal to zero, and the second is when $m$ is equal to an integer.

$$
\begin{gathered}
J_{0}(x)=\sum_{m=0}^{\infty}(-1)^{m}\left(\frac{\left(\frac{x}{2}\right)^{2 m}}{m !^{2}}\right) \text { for } m=0 \\
J_{m}(x)=\sum_{n=0}^{\infty}(-1)^{m}\left(\frac{\left(\frac{x}{2}\right)^{2 m+n}}{(m !)(m+n) !}\right) \text { for } n=\text { integers }
\end{gathered}
$$

It will become apparent that the arbitrary variable $m$ will describe the number of radial lobes in a mode and another arbitrary variable, $n$, will describe the number of circumferential lobes. The pressure field of the wave is described by the Bessel function where the number of radial lobes, $m$, corresponds to the number of the Bessel function needed to model it. The derivative of the Bessel function will give various zero crossings which here are labeled as the circumferential lobe number, $n$. For every zero crossing of the derivative, another circumferential node will be present in mode shape. Each time that a derivative of a Bessel function is zero, these are the only valid solutions of the Bessel 
function that can exist in the duct. The figure below illustrates a pressure field of the mode shapes based on the number of $m$, and $n$.

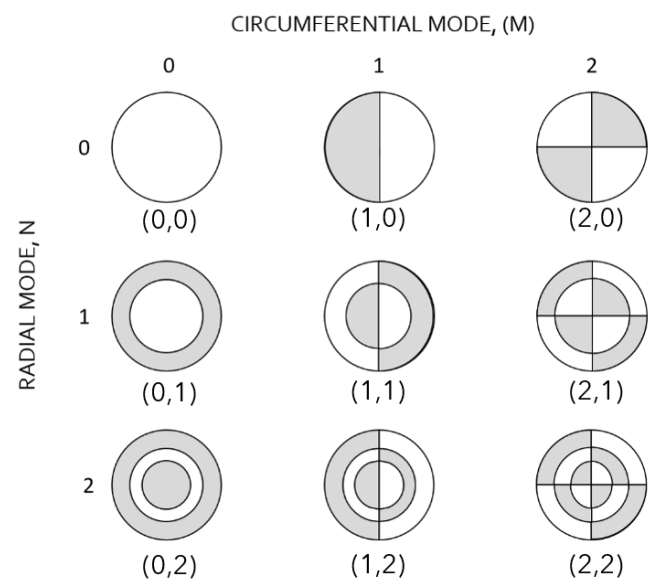

Fig. 7 Circular duct mode shapes generated from Bessel functions. $\mathrm{M}$ is the number of circumferential modes and $\mathrm{N}$ is the number of radial modes. This matrix shows the pressure fluctuations of the gray and white regions [28].

As show in the figure, the $(0,0)$ mode is constant across the cross section of the duct. This is the planar mode. The non-planar modes are the mode shapes that are more complex and have moving components to them. The cut off frequency, or cut-on frequency as it is sometimes known, is the frequency where the sound transitions from being planar to non-planar. The cut-on frequency equation states that

$$
f_{c}=\frac{1.841 c}{2 \pi a}
$$

Where $f_{c}$ is the cut on frequency, $\mathrm{c}$ is the speed of sound, and a is the radius of the duct in meters. For this 6-inch duct, the cut on frequency calculates to $1318 \mathrm{~Hz}$. It is important to note that 1.841 is the zero crossing of the first mode. These zero crossings of Bessel functions can be found in a lookup table [27]. The issue with CNT speakers is that the sound is projected equally along the entire speaker at every angle in the duct. This creates problems because to cancel the complicated mode shapes, the pressure around the duct needs to vary. There is still hope in cancelling modes that occur beyond the cut on frequency. Every pressure wave that occurs in the duct is a superposition of all the modes. This means that the $(0,0)$ mode will still exist in the duct above the cut on frequency, however, it will not carry all of the modal energy. It is still theoretical possible to cancel the planar part of the non-planar mode, but not the mode shape itself. To do this, one would need multiple sections of CNT speakers surrounding the duct that could be independently controlled to create different pressure shapes. This is possible to do with a CNT speaker, but this goes beyond the scope of what the speaker in this paper proposes. 


\subsection{How Non-Plane Waves Are Generated}

To get non-planar waves in a duct, a few things need to be true. The frequency of the source needs to generate waves that are small enough that the duct can support the mode shape. If the wave size is too large, they will propagate as plane waves. At low frequencies, this is typically the case unless the duct has an incredibly large cross-sectional area. The second portion is that the disturbance needs to be off center of the duct. If the noise source is symmetrically aligned in the duct, it will not be able to generate circumferential mode shapes, since the pressure will never be split. So, to achieve true non-planar waves in the duct, the disturbance needs to be off-axis. An example of this was crafted in the Falstad ripple tank simulator [28]. The simulations below illustrate the different cases for which this happens. In the simulations, the small dot is a point source and radiates sound at a given frequency. The two hard walls in the simulation act as a waveguide while sound travels down it.



Fig. 8 A plane wave is sent through a hard wall duct from the point source on the left. The pipe duct is small enough and the frequency is low enough that only plane waves can propagate [28].

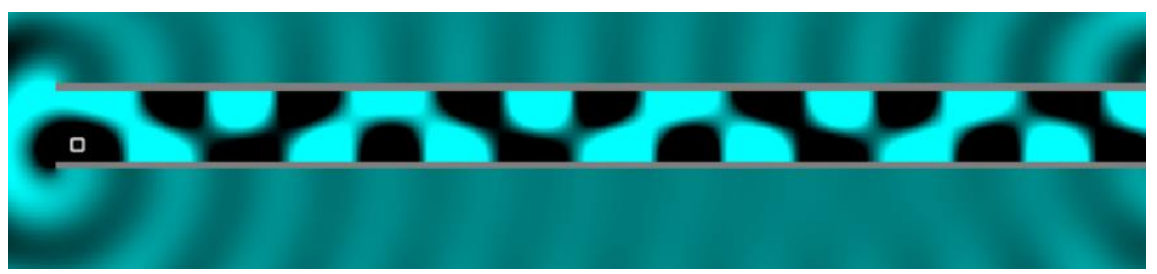

Fig. 9 An off-axis point source (left dot) is now allowing the $(1,0)$ mode to propagate in the pipe. The pressure is no longer planar over time [28].

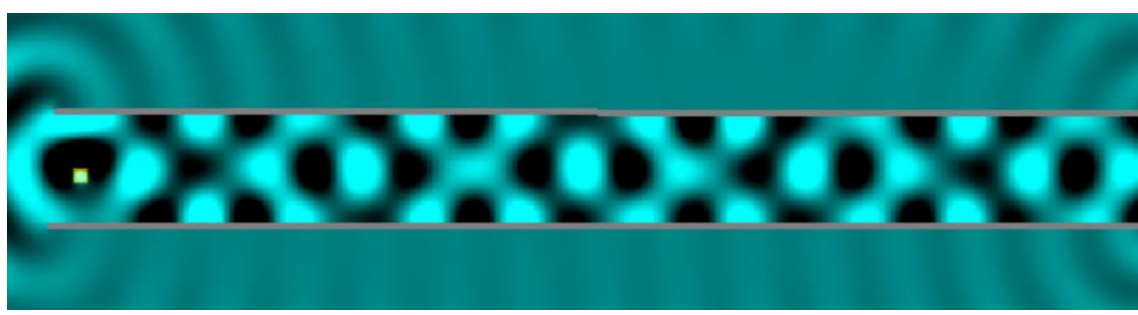

Fig. 10 The frequency and duct diameter have been changed which allows the $(0,1)$ mode to form. The "donut" mode is the alternating pressures down the duct caused by parameter changes, even though the point source is centered [28]. 


\section{Planar Wave Cancellation}

A planar wave is a wave that propagates in one direction [27]. It is a longitudinal wave based on a modified version of the wave equation. The wave equation dictates that a wave is dependent of acceleration in space and acceleration in time. Since the shape of the wave is the same shape as the cross-section of the waveguide, it will propagate down the pipe with even pressure along the geometry of the waveguide. A waveguide is simply a structure with a rigid boundary, such as a duct, that allows a wave to travel with minimal loss. A waveguide typically has a standing wave component caused by reflections down the pipe, and a progressing component that is the direction of desired sound travel [27].

Cancellation of planar modes is very simply done mathematically. The driving principle is for the incident noise wave from the fan that is travelling down the duct to meet with the same wave that is $180^{\circ}$ out of phase with the noise source. When these two noise sources meet, they will create destructive interference that will cancel the sound pressure of the resulting wave.

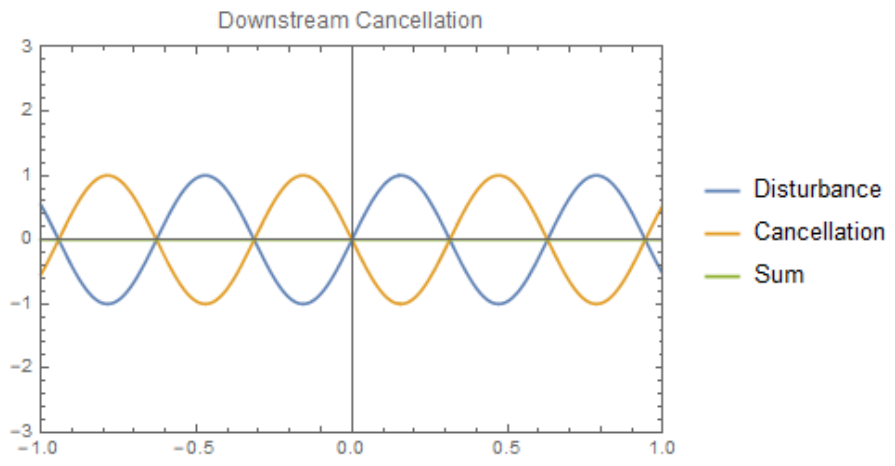

Fig. 11 Downstream cancellation visualization. The fan disturbance (blue) and the cancellation signal (orange) will meet and cause a destructive interference sum (green) between the two signals.

Travelling upstream of the disturbance, a standing wave will form from the two signals traveling in opposite directions. There will be both constructive and destructive interference, which will cause a standing wave in the pipe to form. The figure below shows a standing wave. 


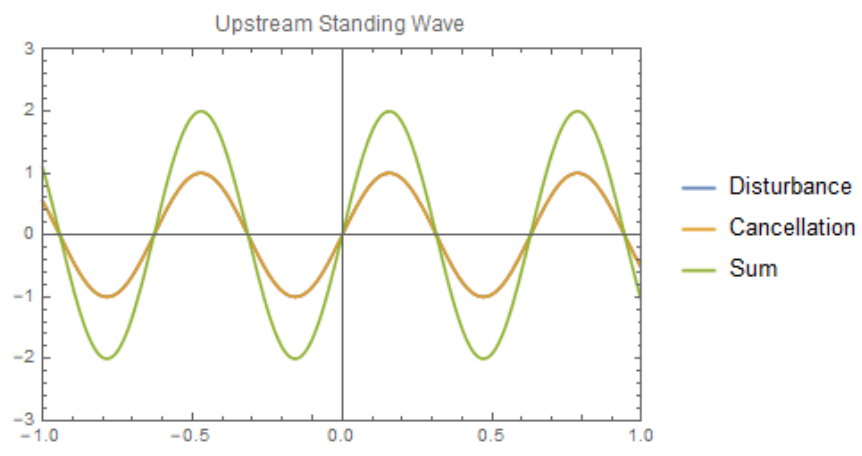

Fig. 12 The wave that travels toward the noise disturbance. It creates a standing wave which adds when the cancellation signal and disturbance signal meet.

The primary noise source is caused by the fan which is a function of the number of blades spinning, and the speed at which they rotate. This is referred to as the blade pass frequency and can be calculated using this simple equation which states that

$$
f_{\text {blade }}=N_{b} \frac{R P M}{60} \mathrm{~Hz}
$$

Where $f_{\text {blade }}$ is the blade pass frequency of the fan found by using the number of blades, $N_{b}$, multiplied by the speed of the fan, RPM, divided by 60 cycles/sec. For this fan, 8 blades were used. In the ANC system, the tachometer measures the number of times a single blade spins and from there creates a pulse train of RPM to gauge the speed of the fan. Based on this, specific orders can be selected to be cancelled. Most of the tonal noise is the frequency spectra of the fan is dictated by the fundamental of the blade pass frequency, and harmonic components. This makes cancellation of these planar tones simple since a waveform that accounts for dominant tonal harmonics can be selected and played through the duct at a $180^{\circ}$ offset to create the destructive interference.

For the best chance of cancelling planar waves, certain positions of the microphone, noise source, and cancelling speaker need to be considered. It was found through preliminary testing that the best solution to cancelling plane waves would be to have the cancelling source after the noise source. The distance between the two sources is arbitrary - the only component that matters is if the waves have enough time to form plane waves as they travel down the duct. The initial shape of the wave at the noise and cancellation sources can be messy and contain unstable higher order modes that are hard to cancel and exponentially decay over time; however, the waves will eventually stabilize given enough time and travel distance. This is one advantage of using CNT speakers as opposed to traditional ones; the coaxial CNT speakers can create pure plane waves at the noise source. Using this advantage, the error microphone of the ANC system is best placed at the opening of the duct, as far away possible from the cancellation and disturbance. This will give the waves enough time to form stable plane waves that the ANC system can cancel. 


\section{CNT Construction and Design}

\subsection{Materials}

For this project, many different materials were considered. As mentioned earlier, CNT speakers can reach extreme temperatures. A material needed to be found that was flexible, non-conductive, and robust at high temperatures. Furthermore, the material needed to not impede the sound produced by the speaker as this would drive up the power consumption to make the speaker loud. After many years of different materials researched in the lab, fellow researchers Walker Nelson and Steven Senczyszyn came across a PTFE coated woven fiberglass fabric that was shown to work for this application [29]. This material is flexible, porous, and can withstand long-term high temperatures. The porosity allows the nanotubes to be safely encased in two sheets of the PTFE material while allowing sound to travel. The PTFE coated fiberglass (PTFE FG) is 0.003 " inches thick and can withstand a maximum temperature of $500^{\circ} \mathrm{F}$. With these properties, it makes it an ideal candidate for the CNT backing material.

To create an electrical circuit, copper tape was used. This allows for the CNT to be connected in special electrical configurations while also allowing the speaker to maintain its flexibility. The copper tape is laid in a pattern that optimizes the resistance of the speaker. Simple wire was soldered to the copper tape to allow for the speaker to receive a signal. It was found that a larger gauge was needed for long term/high power use, but for the purposes of the test, 18-gauge wire was acceptable.

Multiwalled CNT (MW CNT) that was created via chemical vapor deposition was used as the primary source of CNT for this project. It is important to note that single walled nanotubes will not work to create a CNT speaker in this capacity.

\subsection{Design}

To design the resistance and size of the speaker, a MATLAB code was created to give the user an idea of the size and spacing of the copper tape electrodes. It is important to note that CNTs act a resistor element in a circuit, so the amount of CNT will change the resistance of the speaker. The program works primarily with the assumption that 1 square area of CNT has a theoretical resistance of $150 \Omega$ for 5 layers of CNT. The program will ask the user what the dimension of the cylindrical duct are - in this case, the duct was 6" in diameter. It will also allow for the user to specify their desired resistance. It will then calculate the number of squares needed to reach that resistance, the number of electrodes needed, and the height of the cylinder required. The following equations demonstrate the electrical calculations:

$$
\text { squares }=\frac{150}{r}
$$


Where $\mathrm{R}$ is the resistance and squares are the number of squares required.

$$
\text { squares per electrode }=\frac{\text { squares }}{\text { arc number }}
$$

Where arc number is the number of electrodes and is iterated in the code from 1 to 10 .

$$
\operatorname{arc~length}=\frac{(\pi * d-(\operatorname{arc} \text { number } * 0.25))}{\operatorname{arc~number}}
$$

Where arc length is the spacing between each electrode, $\mathrm{d}$ is diameter, and 0.25 is the width of the copper tape electrodes used. Lastly, the height is calculated.

$$
\text { height }=\operatorname{arc} \text { length } * \text { squares per electrode }
$$

Height will relate the required dimension that the cylinder needs to be. Using these equations, the dimensions of the speaker and the resistance of the speaker can be chosen. For this project, a 6" diameter was chosen to fit the inside of the pipe. The height of the speaker was 5 inches and the number of electrodes was 8 . This resulted in a resistance of $14 \Omega$.

5 layers of CNT was chosen because too few layers will not produce the same sound output and too many layers did not significantly contribute to a higher sound level. 5 layers is a standard number chosen in lab to make speakers as it will adjust for the best CNT sound output levels. 


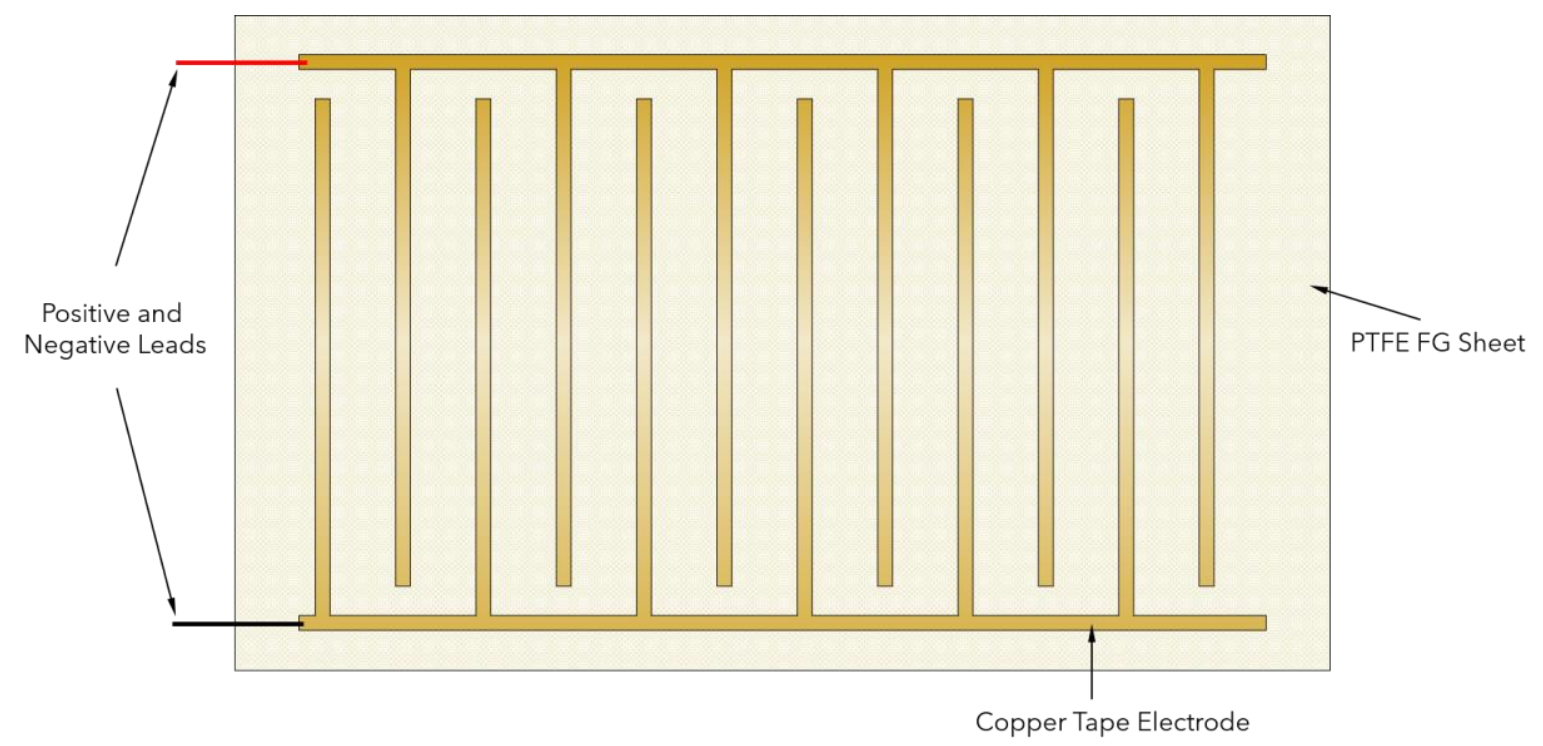

Fig. 13 Copper tape electrode configuration.

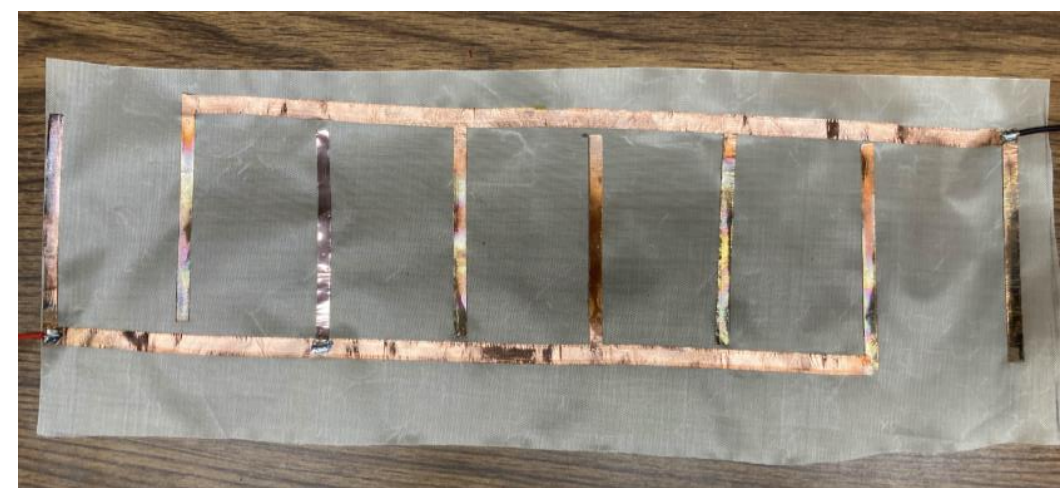

Fig. 14 Electrode pattern created using copper tape and adhered to PTFE FG.

The cost of each individual speaker is based solely on the amount of material needed to create the speaker. In practice, material is wasted in creating the CNT speaker, particularly CNT. This bill of materials provides a current cost breakdown of what it takes to build a single speaker with this design. It does not include the initial startup costs of the speaker. Appendix A provides the entire bill of materials (BOM) for the speaker. The average price for a 6 " speaker is currently $\$ 46.65$. This price could further be brought down by buying material in bulk for large scale production. Table 1 shows the breakdown for the speaker in its current design with 8 electrodes. 
Table 1 . The bill of materials for creating the speaker

\begin{tabular}{|c|c|c|c|c|}
\hline & Price/unit area & Area Used & Tot & 1 Price \\
\hline CNT Film & 0.10 & 376.8 & $\$$ & 39.19 \\
\hline Electrodes & 0.07 & 26.85 & $\$$ & 1.87 \\
\hline PTFE Fiberglass & 0.02 & 237 & $\$$ & 4.16 \\
\hline Adhesive & 0.04 & 15.925 & $\$$ & 0.65 \\
\hline \multirow[t]{2}{*}{ Wire } & 0.11 & 6 & $\$$ & 0.68 \\
\hline & & Total & $\$$ & 46.54 \\
\hline
\end{tabular}

\subsection{Construction}

To build the speaker, the PTFE FG was first cut to size. The electrode pattern was cut out of a sheet of thin copper with adhesive on the back. The adhesive electrode pattern was applied onto one the PTFE FG sheets and solder was applied to any copped electrodes with loose connections. Next, the wires were soldered to either side of the electrodes so that they could be run out of the duct for the speaker to receive a signal. Double sided Kapton tape with adhesive silicon was applied to the electrodes such that when the final top sheet of PTFE FG was applied, it would adhere tightly.

After all of these set up steps had been completed, the CNT wrapping can start. The PTFE FG and electrode sheet was taped down to a 6-inch diameter cylinder. The cylinder has an axle shaft that runs through it connected to a handle. This allows the cylinder to spin in any direction the user desires. The figure below illustrates the speaker on the cylindrical wrap station. Where the two sides of the sheet connect on the cylinder, the speaker was taped with electrical tape leaving a small gap where the sheets connect. This is important for the final step after the wrapping is done. The figure below illustrates some of the major steps involved in making the CNT speaker. 


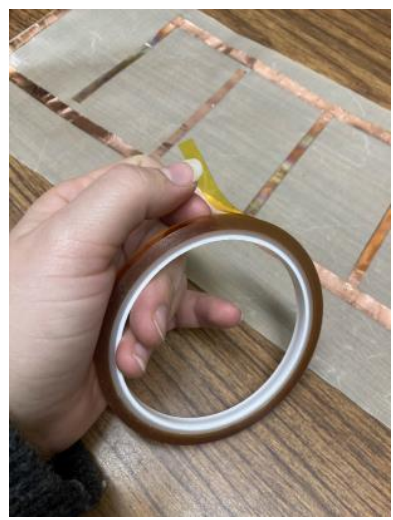

Step 2 of making the speaker is to use Kapton tape on the joints

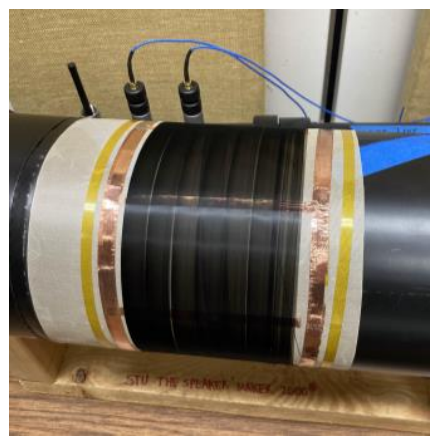

Step 5 is to completely wrap 5 layers of CNT across the speaker

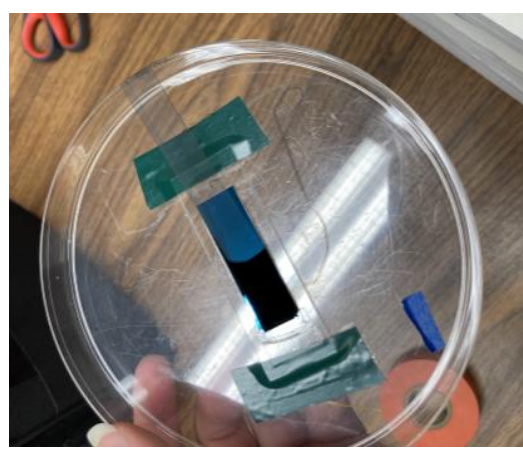

Step 3 is to pull a new or used CNT forest into a film to be used on the speaker

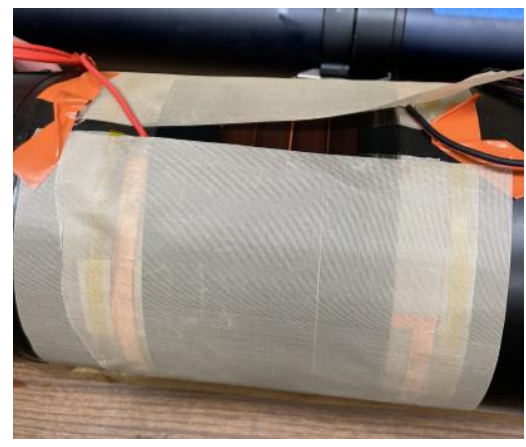

Step 6 shows that the top layer of PTFE needs to be ahered to the Kapton tape

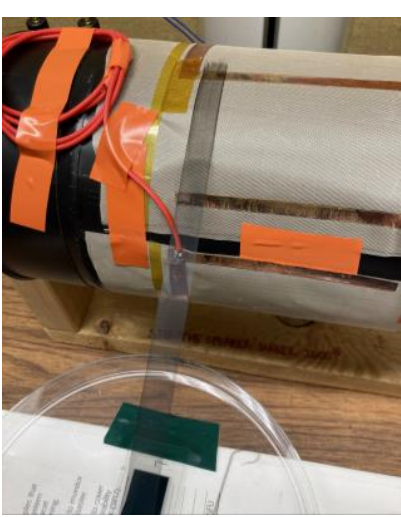

Step 4 is to gently pull the CNT across the electrodes and onto the spinning mechanism

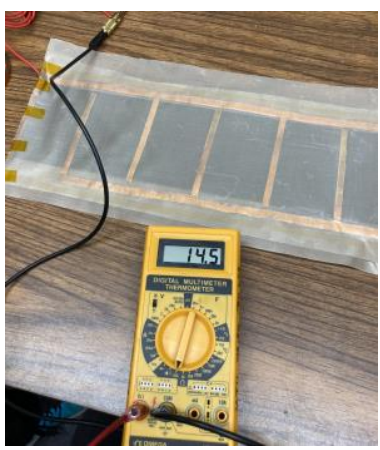

Step 7 illustrates a completed speaker being tested after the build. The resistance was $14.5 \Omega$

Fig. 15 A quick guide to constructing a CNT speaker.

The trickiest and most important step is the CNT wrap. First CNT is pulled from a forest, forming a long thin film. The film is then laid at the far-left side of the speaker. Slowly, the cylinder is turned which pulls the CNT forest further. This process is repeated until 5 layers of CNT have been placed in one section of the speaker. The CNT is then slowly moved to the right of where the wrapping was done, causing the CNT to become pulled at an angle. The cylinder will spin again until the CNT is wrapping in a new section of the speaker. This process repeats until the entire speaker is wrapped. Then, CNT film is "cut" from the speaker using denatured alcohol and a knife. The denatured alcohol severs the bonds in the CNT film and the left-over film is carefully cut. The CNT is then covered and put away to ensure no damage or dust comes to it. CNT is also carefully cut at the point 
where the two sides of the speaker join on the cylinder. The excess CNT is scraped off to ensure that none touch any of the electrodes, which would result in shorting the speaker.

The last step is to apply the top PTFE FG sheet such that the CNT is encased in two layers of it. The sheet is taken off the cylinder wrapping system so that it lays flat on the table. The top sheet is carefully aligned and applied first by hand, then the cylinder is removed and used to roll over the speaker to apply extra uniform force. After this, a flexible CNT speaker is achieved.

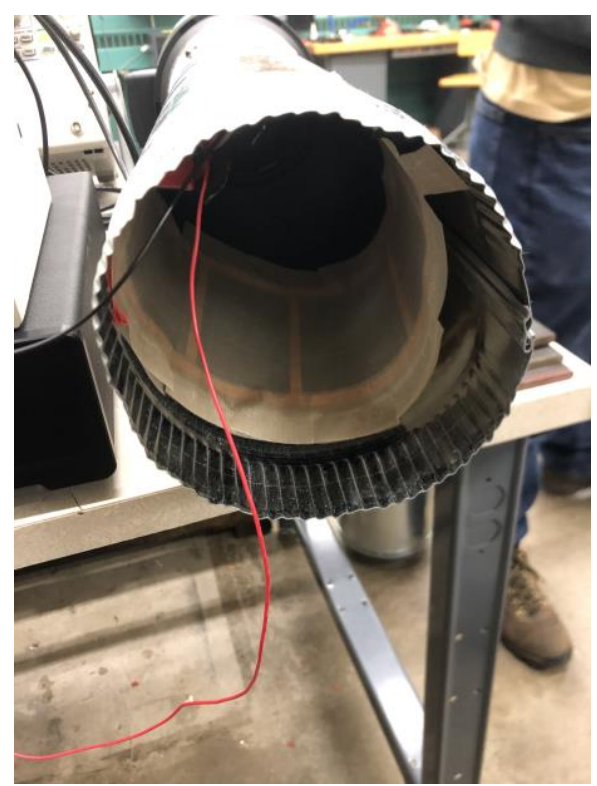

Fig. 16 CNT speaker taped into the duct system. The speaker is not taped down all the way to better show its flexibility in the speaker. 


\section{Methods and Materials}

\subsection{Planar Wave Cancellation Setup}

\subsubsection{DAQ Setup}

For the purposes of testing, an LMS SCADAS XS was used as a data acquisition system. There were 3 channels collected: The error microphone, the drive signal sent to the speaker, and the tachometer channel. The tables below shows the data acquisition parameters used in collecting data for planar wave cancellation.

Table 2. LMS Data Acquisition Hardware Parameters

\begin{tabular}{|c|c|c|c|c|}
\hline Channel & Model & Sensitivity & Power Coupling & HW Range \\
\hline Microphone & PCBA24 & $101.8 \mathrm{mV} / \mathrm{Pa}$ & AC & $3.16 \mathrm{~V}$ \\
\hline Tachometer & Modal Shop Laser Tach & $1000 \mathrm{mV} / \mathrm{V}$ & DC & $2.5 \mathrm{~V}$ \\
\hline Drive Signal & -- & $1000 \mathrm{mV} / \mathrm{V}$ & AC & $10 \mathrm{~V}$ \\
\hline
\end{tabular}

Table 3. Planar Wave Testing Data Acquisition Sampling Parameters.

\begin{tabular}{|c|c|}
\hline Sampling Frequency (Hz) & 0.5 \\
\hline Resolution (Hz) & 51200 \\
\hline Span (Hz) & 2000 \\
\hline Number of Averages & 51 \\
\hline Recording Time (s) & 10 \\
\hline
\end{tabular}

Instead of using a tachometer channel on the data acquisition system, a virtual channel was setup in LMS that the following parameters: cross over level -0.5, 1 pulse per revolution, a negative slope detection, and upper and lower tolerance of 0 , and an upsampling factor of 2. These parameters captured pulse train data from the tachometer and converted it to a useable signal in real time, without the need for a tachometer channel. This was advantageous as the SCADAS unit did not include a tachometer channel and the tachometer data was needed for trigger settings and to ensure that the tachometer was functioning appropriately. 


\subsubsection{Hardware Setup}

The rest of the setup used a DSonic amplifier, a PCB model 482A 16 signal conditioner, an Nx4 Preamp, a Larson Davis Cal 200, a handmade amplitude modulator breadboard, and a Vivosun 6" fan with 8 blades. The setup is described in the figures below:

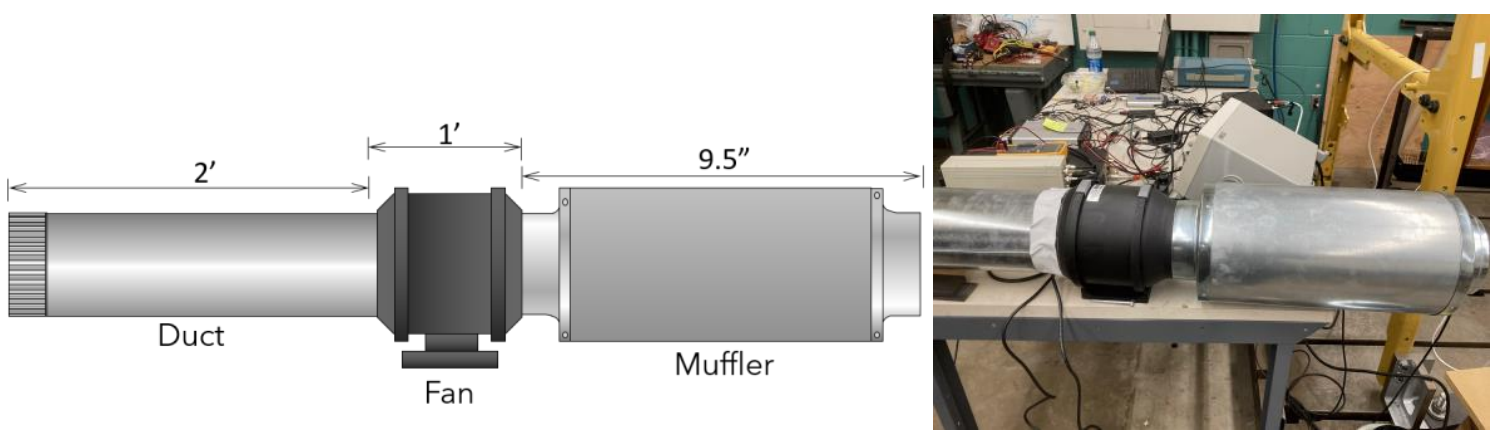

Fig. 17 A side profile of duct setup with their lengths

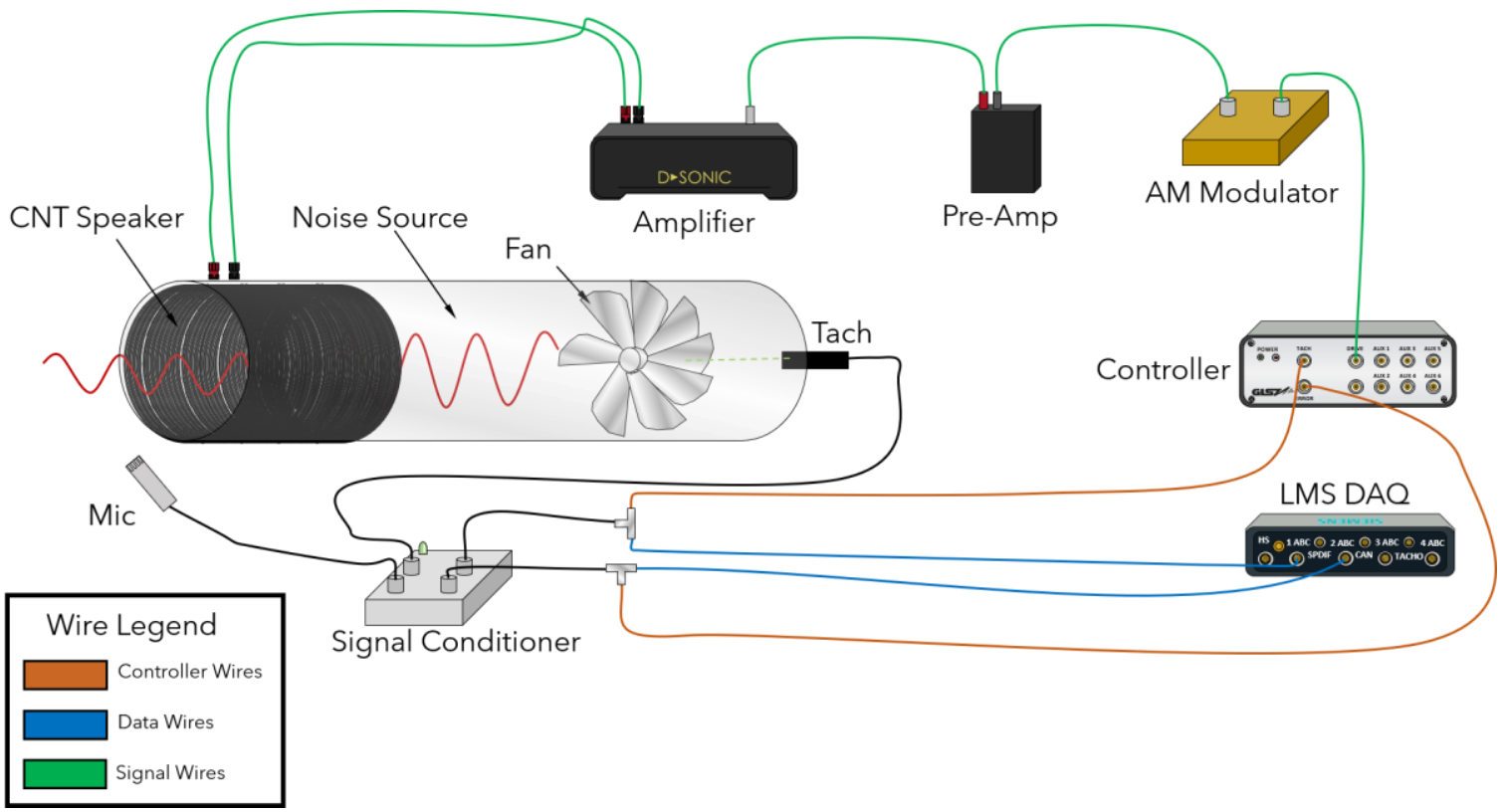

Fig. 18 The planar wave cancellation setup with the 8-blade fan installed in the rear. The GLSV measurement system is connected to the error microphone and sends a drive signal to the CNT speaker to cancel noise from the fan.

The setup allows the blade pass frequency that is generated by the fan to be measured by a tachometer. The tach signal is split and is sent to both the GLSV ANC controller and the LMS DAQ. The ANC controller sends the signal for cancellation first to the amplitude modulator (AM). This step is important since CNTs have a unique property of doubling the output frequency of the speaker. The amplitude modulator will send the tone at a $20 \mathrm{KHz}$ carrier frequency. The signal is then sent to the Pre-amp to increase the gain of the signal before being sent to the amplifier. The signal is then sent to the amplifier which will provide power to the CNT speaker. The CNT speaker outputs a $180^{\circ}$ out of 
phase signal to attempt to cancel the speaker noise. The microphone is positioned at the end of the duct with a wind screen on it, out of the path of flow. The microphone will measure the sound pressure at the end of the duct and send the signal to the signal conditioner. The signal is sent to a signal conditioner to both power the microphone and split the microphone signal between the LMS DAQ and the ANC error control. The ANC error control signal feeds back into the narrowband feed forward FXLMS algorithm to constantly correct for any changes in the tach signal. The tachometer is also powered via the signal conditioner. The image below shows the actual test setup used.
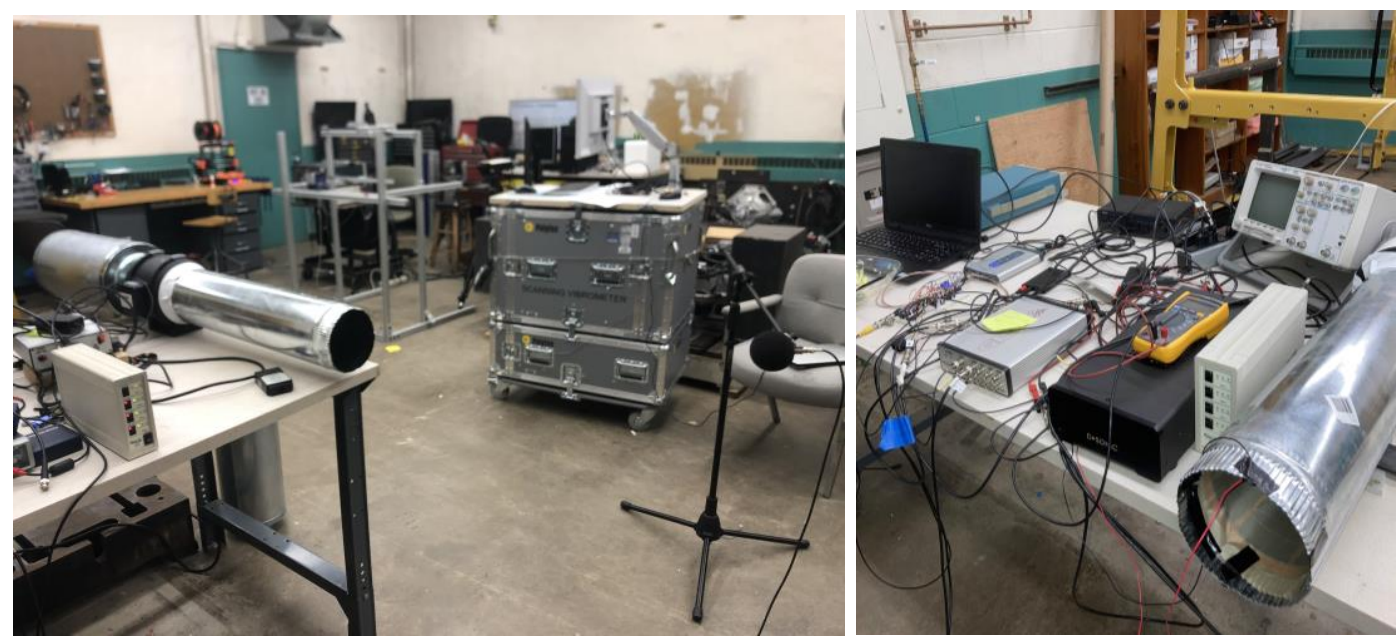

Fig. 19 Lab setup for planar wave cancellation testing.

\subsubsection{ANC Setup}

Lastly, the GLSV ANC Controller needed to be configured and setup before cancellation began. This was a two-step process: step 1 is to characterize the speaker microphone setup. This is achieved by playing white noise through the speaker and measuring the transfer function at the microphone position. The settings entered for cancellation on this setup can found in the figure below. 




Fig. 20 The user interface for the GLSV ANC controller.

The "Ramp Time" is the amount of time that the controller will transition from off to on over the course of a given time (in this instance, 1 second). This is to prevent damage to the speaker. The "Fade Time" is the transition time to power off. "Start Freq" is the starting frequency that the ANC will characterize over. The "End Freq" is the upper limit of frequency that the speaker will characterize over. So in the above example, the CNT speaker will play white noise from 100 to $1000 \mathrm{~Hz}$ to characterize the speaker. The "Number of Frequencies" is the number of data points between the start and end frequencies. This parameter controls the frequency resolution (in this case, $9 \mathrm{~Hz}$ ). 101 number of frequencies were chosen because the phase signal changes slow enough that a bandwidth of $9 \mathrm{~Hz}$ can accurately capture the signal while making the characterization time and memory use appropriate. "Orders" is the list of orders that the user desires to cancel. This can be any number include decimal values. For example, if a user wanted to cancel at the $2.5^{\text {th }}$ order, they could type in 2.5 in the order cancellation and the system would cancel. The "Stepsize" is the rate at which the FXLMS algorithm converges. The step size is used in calculating the gradient descent portion of the algorithm which attempts to converge on the optimal wave parameters. This step is especially important in ANC cancellation because if the step size is too small, it will take a longer time for the signal to converge as it slowly steps down the gradient to the optimum. If the step size is too large, the optimum can be overshot, and the system will bounce unstably. Step size must be defined as a list for ever order attempting to be cancelled.

"Trigger Threshold" is the voltage threshold from the tachometer signal that looks for the rising edge in the pulse train. This parameter is used to get an accurate tracked signal from the tach. "Trigger Hysteresis" is used for when there is accidental noise introduced in the tach signal. It will shift the rising edge threshold up by a specific value and shift the falling edge down by the same threshold. This ensures that noise does not accidentally trigger the threshold. For these purposes, the Trigger Hysteresis was set to 0.10 as this was found during testing to be the best setting. 
The "Frequency Generator Threshold" are inherent properties of the algorithm and should not change. The defaults were used for this testing as recommended by the manufacturer. Inside the software is a phase lock loop that attempts to lock the tachometer signal of the system which contains two properties: "Frequency Generator Proportional Gain", and "“Frequency Generator Derivative Gain". These are the closed loop parameters of the digital phase locked loop and should not be altered as well. "Frequency Generator Resting Frequency" is the initial guess that the phase locked loop takes to finding the speed of the tachometer. It should be set to the mean value that is expected of the tach loop. For the purposes of testing, this was $42 \mathrm{~Hz}$, as the average RPM of the fan was $2520 \mathrm{rev} / \mathrm{min}$. It is important to note that the farther away the tach signal is from the initial guess, the slower the convergence will be. The phase locked loop can only converge to a tach signal that is twice as fast or twice as slow as the initial guess.

"Decimation Rate" is the number of samples that the system discards when processing. This is done because performing the processing at the default rate of 48,000 $\mathrm{Hz}$ can negatively impact the CPU utilization of the system. This is important because for every order that needs to be cancelled, the number of computations is effectively doubled. By decimating, it allows the system to cancel more orders. For this, the decimation rate of 2 was chosen because it is the minimum needed to handle multiple orders and increased the efficiency while maintaining accuracy.

In "Characterization Settings", "Decimation Rate" is the same as described above. To decimate here, it has the added benefit of concentrating the energy in the bandwidth that is desired. This can help increase the coherence for unneeded frequencies that are outside of the target range. "Gain" controls the amplitude of the white noise signal that is played through the speaker. The gain was left at one and amplitudes were toggled using the Pre-Amp for testing. "Measurement Iterations" is the number of averages that the coherence is calculated over while characterizing the system. Here it was left to the default of 10. The rest of the parameters shown in the figures have been described in some capacity and were typically left at their default values as this proved to be effective in testing. After all the hardware and software was setup, testing on planar wave cancellation began.

\subsubsection{Extended Planar Wave Cancellation}

To measure whether or not planar waves would continue to cancel over time, the DAQ was altered to measure over different time increments. In the stability measurements, 351 averages were used over a period of 70 seconds. Few of the other stability measurements also contained a prolong number of averages and data recording time. 


\subsection{Non-Planar Wave Cancellation Setup}

\subsubsection{DAQ Setup}

The DAQ setup for non-planar wave cancellation testing was similar to the planar cancellation with some minor differences. The table below shows all testing parameters for non-planar wave cancellation.

Table 4. LMS Data Acquisition Hardware Parameters

\begin{tabular}{|c|c|c|c|c|}
\hline Channel & Model & Sensitivity & Power Coupling & HW Range \\
\hline Microphone & PCBA24 & $103.1 \mathrm{mV} / \mathrm{Pa}$ & AC & $3.16 \mathrm{~V}$ \\
\hline Tachometer & Modal Shop Laser Tach & $1000 \mathrm{mV} / \mathrm{V}$ & DC & $2.5 \mathrm{~V}$ \\
\hline Drive Signal & -- & $1000 \mathrm{mV} / \mathrm{V}$ & AC & $10 \mathrm{~V}$ \\
\hline Microphone & PCBA24 & $6.41 \mathrm{mV} / \mathrm{Pa}$ & ICP & $10 \mathrm{~V}$ \\
\hline
\end{tabular}

Table 5. Planar Wave Testing Data Acquisition Sampling Parameters.

\begin{tabular}{|c|c|}
\hline Sampling Frequency (Hz) & 0.5 \\
\hline Resolution (Hz) & 51200 \\
\hline Span (Hz) & 2000 \\
\hline Number of Averages & 51 \\
\hline Recording Time (s) & 10 \\
\hline
\end{tabular}




\subsubsection{Hardware Setup}

The important difference in this setup was that an ICP powered microphone was added downstream of the pipe. The figure below illustrates the test setup.

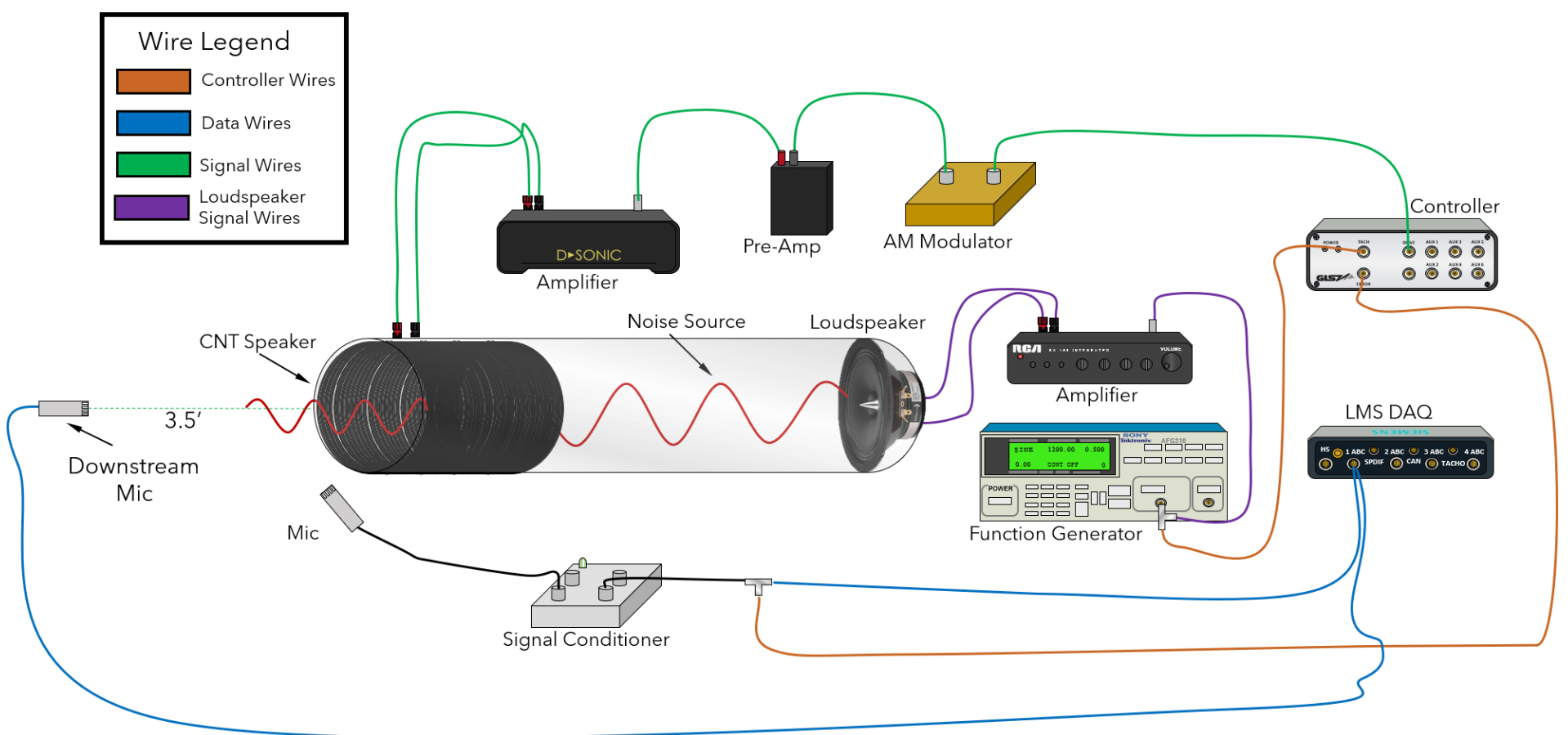

Fig. 21 The non-planar wave cancellation. An addition of a frequency generator, amplifier, and loudspeaker in lieu of a fan.
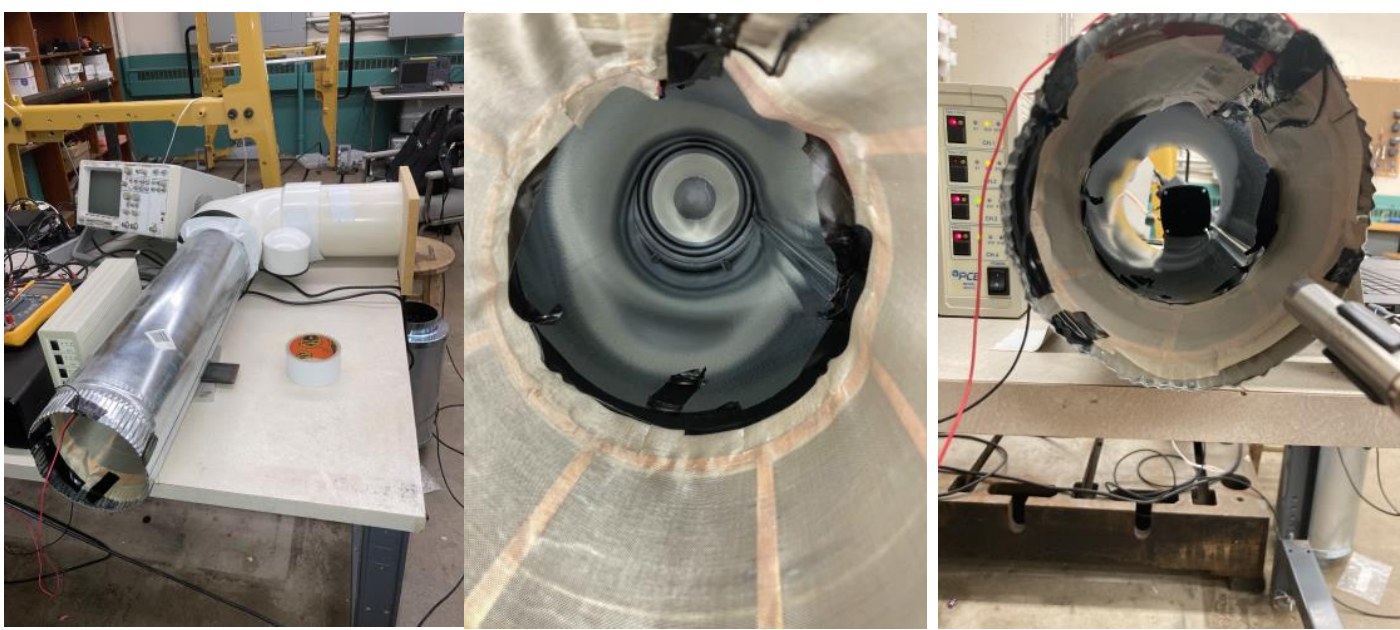

Fig. 22 The inline test setup (right) and non-inline test setup (left). 
For non-planar testing, the sound in the duct needed to reach a frequency beyond the cutoff frequency of $1,317 \mathrm{~Hz}$ (see section 4 for explanation). It was found during testing that the fan did not spin at a fast-enough rate to generate sound data above this range. Because of this, a loudspeaker replaced the fan in the duct. A Sony Tektronik AFG310 frequency generator was used to send a signal to an RCA SA155 Integrated amplifier. This amplifier powered two different types of loudspeakers: one that was centered and was 6inch which completely encapsulated the duct, and a smaller 3-inch speaker that was placed off center of the duct. The microphone was placed at 7 pipe diameters downstream of the speaker to measure the plane wave cancellation.

\subsubsection{ANC Setup}

The ANC setup had some fundamental differences for non-planar testing. The start and end frequencies were 1000 to $2000 \mathrm{~Hz}$, respectively. This would characterize the speaker in the range that was needed. The idea was to test frequencies right below the cut off frequency, at the cutoff frequency, and above the cutoff frequency. The new characterization range provided the ability to test these frequencies. The frequency generator resting frequency initially remained the same as it was found that it still surprisingly worked on testing. This was changed later to $1000 \mathrm{~Hz}$ to better reach the output signal of the loudspeaker which was acting as tachometer. 


\subsection{Accelerated Life Testing Setup}

To do a small-scale test on accelerated life testing, a new CNT speaker was built using the same exact parameters for consistency. Here, the CNT speaker was taped in the duct again, but this time the fan was replaced in the back of the pipe. The fan was set to run at its highest velocity to get the most amount of cooling on the CNT, and to simulate real life test conditions. To measure the quality of the speaker over time, 3 different metrics were collected: voltage, current, and sound pressure near the speaker. The voltage and current were collected on two different NI virtual benches using a LabView program. The parameters were setup such that the NI system would take a voltage and current reading every minute of testing. To collect sound data, an LMS SCADAS X setup was used which contained only 1 ICP powered microphone. Fig. 23 highlights the testing setup used.

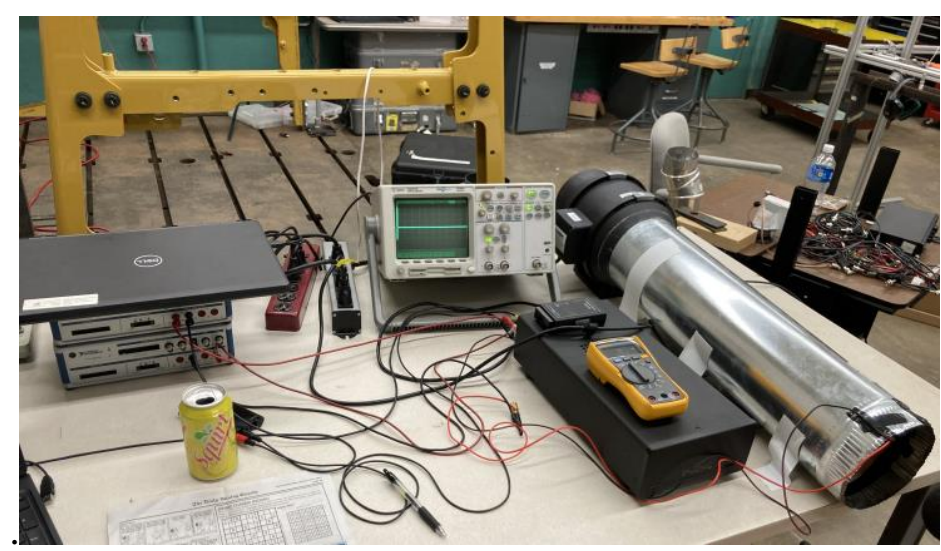

Fig. 23 A picture of the accelerated life test setup

The microphone was at the direct outlet of the duct, off center, to capture the sound without the wind energy. This was manually operated to take a 10 second sound measurement every 10 minutes. The 10-minute resolution was chosen to ease the analysis of sound data, while maintaining enough samples to catch the sound output over the life test. The table below shows the DAQ parameters.

Table 6. LMS Data Acquisition Hardware Parameters

\begin{tabular}{|c|c|c|c|c|}
\hline Channel & Model & Sensitivity & Power Coupling & HW Range \\
\hline Microphone & PCBA24 & $10.2 \mathrm{mV} / \mathrm{Pa}$ & ICP & $10 \mathrm{~V}$ \\
\hline
\end{tabular}

Table 7. Accelerated Life Testing Acquisition Sampling Parameters.

\begin{tabular}{|c|c|}
\hline Sampling Frequency (Hz) & 0.5 \\
\hline Resolution $(\mathrm{Hz})$ & 51200 \\
\hline Span (Hz) & 2000 \\
\hline Number of Averages & 51 \\
\hline Recording Time (s) & 10 \\
\hline
\end{tabular}


To heat cycle the CNT, a frequency of $20 \mathrm{KHz}$ was chosen as this is the highest frequency that the DAQ could record while allowing the CNT the maximum amount of heat cycles. Every cycle/second in a sound wave is a heat cycle/second in a CNT speaker. It is important to note that for this testing, the amplitude modulator was removed. This is because to generate a tone, a frequency generator was used to output a $10 \mathrm{KHz}$ signal. The nonlinear doubling effect of the speaker caused a sound output at $20 \mathrm{KHz}$. Due to time restrictions, the life test only commenced for 180 minutes. Previous life testing data on other projects were referenced though in the results section. 


\section{Results}

\subsection{Fan Characterization}

Before any testing of the ANC system could be done, the frequency content of the fan had to be accessed. The current setup is for cancellation of narrowband tonal noise, not broadband noise. Once the primary noise contributors were established, the correct orders to be cancelled could be chosen. This was done by doing an order tracking test and testing the fan at the 3 primary speeds: low, medium, and high. As shown in figure 24 , the $8^{\text {th }}$ order is the one of the most prominent orders in the data. This corresponds to the 8 fan blades that are present. It can be expected that most of the tonal sound energy is dominated by the interaction of the fan blades. It can be seen in the figure that there is a resonance that occurs at $360 \mathrm{~Hz}$ that is present in the data at every RPM. This may correlate to electronic noise, but the cause was not determined. It is also dominant in the frequency spectra when measuring the fan. To overcome this, this $360 \mathrm{~Hz}$ frequency was filtered out for sound level comparison as it was not a cancellable tone.

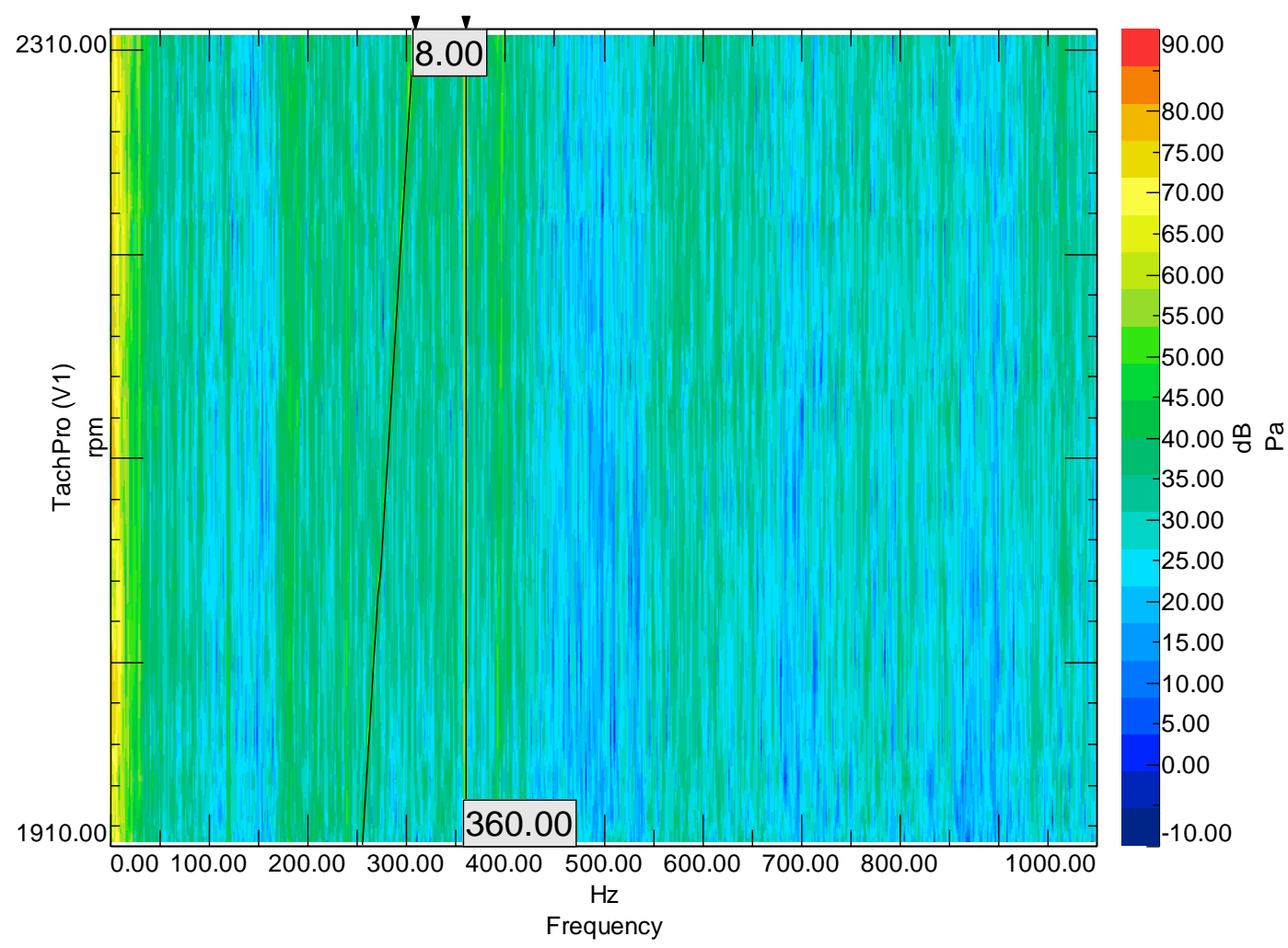

Fig. 24 Order tracking sound data of the 8-bladed fan 


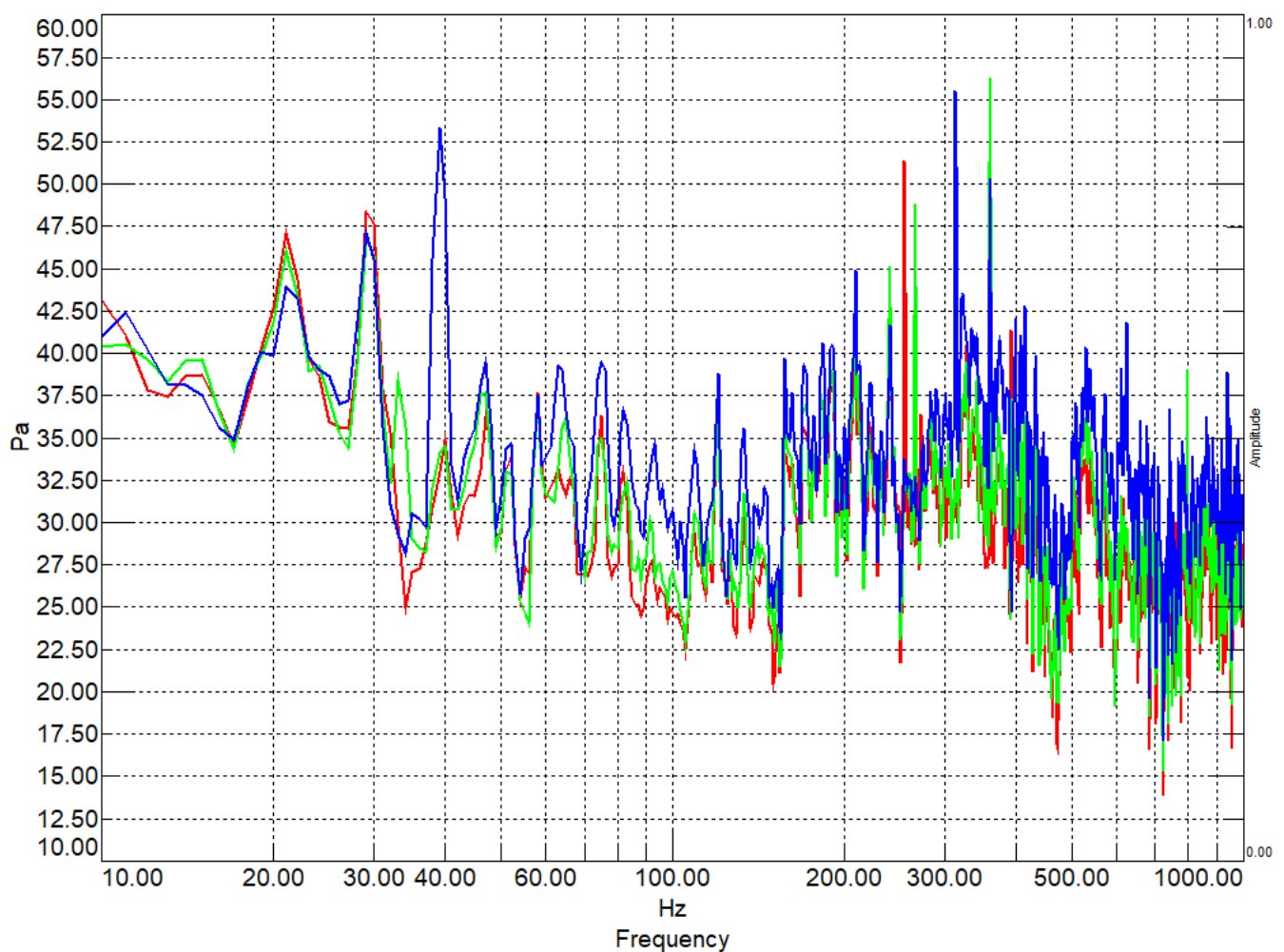

Fig. 25 Frequency spectra of the low (red), medium (green), and high (blue) settings on the fan.

To better access which orders are cancellable, the spectra of highest setting was analyzed, as this was the setting that would be used for testing. It is shown in figure 25 that the blade pass frequency, $314 \mathrm{~Hz}$, is the highest tone in the spectrum. Similarly, the next highest tones are harmonics of the blade pass frequency, as shown by the alignment of the harmonic cursor with the next highest peaks. This makes cancellation easy to perform on this fan because most of the sound energy is tied to the blade pass frequency. The only exception is the $360 \mathrm{~Hz}$ tone which contributed above the noise floor of the data. With this characterization done, it was then determined that the fan orders that need to be canceled were the $8^{\text {th }}$ and $16^{\text {th }}$ orders. Only a maximum of 2 orders were calculated at a time due to the restrictions on the hardware of the ANC. 




Fig. 26 Order tracking done on the fan with a microphone situated 1 meter away at a $45^{\circ}$ angle away from the flow path. 


\subsection{Planar Wave Cancellation}

When cancelling planar waves, the transfer function of the ANC speaker and microphone system needed to be analyzed. As shown in figure 27, the initial planar testing up to $1000 \mathrm{~Hz}$ showed good coherence at the frequencies of interest $(314 \mathrm{~Hz})$. This data also illustrated that the system would not be able to cancel the fundamental frequency $(39.25 \mathrm{~Hz})$ because of the poor coherence in this region. This is expected as lower frequencies are a limit of the ICP conditioning of the microphone.



Fig. 27 Coherence of the ANC system prior to testing

When cancelling for different orders, the main one that was focused on was the $8^{\text {th }}$ order, as it contributed to the highest level of sound pressure of the tonal noise. The figure below shows the cancellation of the $8^{\text {th }}$ order. In these tests, it was consistently possible to get a sound reduction of $20 \mathrm{~dB}$. This is subjectively equivalent to being $4 \mathrm{x}$ as quiet. As shown in the figure below, the red data indicates that the fan was running with cancellation off, and the green data shows the ANC on system. The ANC system successfully cancels the tone at the frequency of interest $(314 \mathrm{~Hz})$ while not adding additional sound energy. Fig. 28 shows the $360 \mathrm{~Hz}$ notch filtered tone represented in an A-weighted 1/3 octave graph. On the far side shows the total sound energy in the system. Despite good tonal cancellation, the total sound level of the fan was not noticeably changed. The total Aweighted sound level for cancellation off was $71.9 \mathrm{~dB}$, while cancellation was $71.4 \mathrm{~dB}$. This indicates that for this fan, tonal cancellation alone was not enough to have a meaningful impact on the total sound level. It can reduce potentially irritating hums and 
tones that may dominate in a fan. On a good note, the CNT speaker appears to cancel at the level with no issues, indicating that is a good system for cancellation.

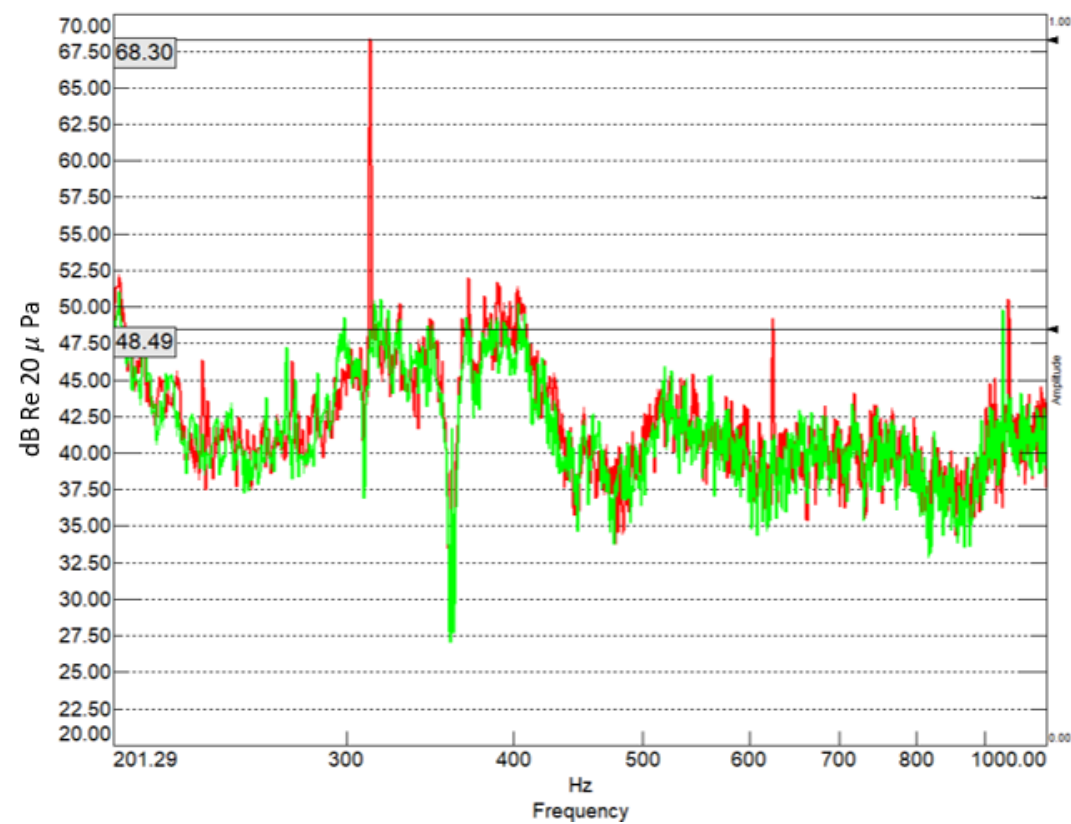

Fig. 28 Sound cancellation of $8^{\text {th }}$ order $(314 \mathrm{~Hz})$ tone. Red data shows cancellation off and green shows cancellation on. The $8^{\text {th }}$ order tone is eliminated.

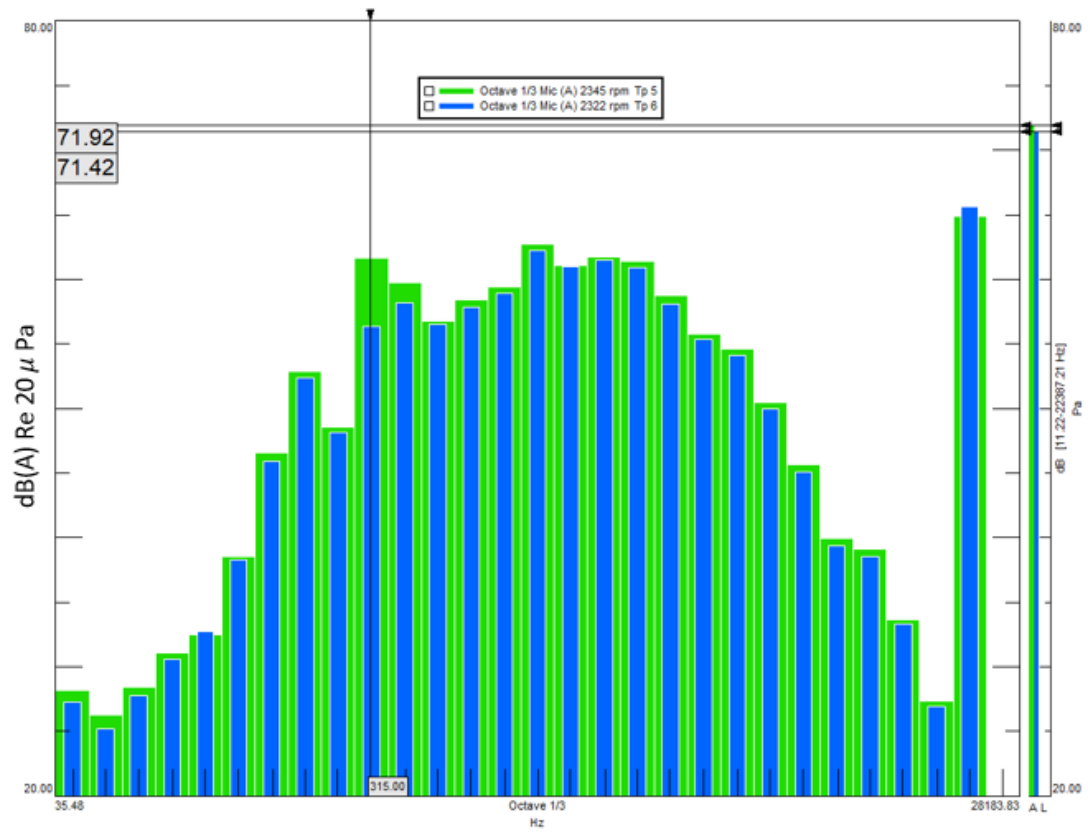

Fig. 29 An A-weighted 1/3 octave representation of the $8^{\text {th }}$ order tone cancellation. Here the green data is the cancellation off and blue data is the cancellation on. It is important to note that the $360 \mathrm{~Hz}$ tone was filtered out in this data. 
Other harmonics in the frequency spectra were also tested to see if the CNT speaker and ANC system could eliminate these tones. Both the $24^{\text {th }}$ and $16^{\text {th }}$ orders were tested as these were the next most dominant tones in the frequency spectrum. Figure 30 shows the $24^{\text {th }}$ order $(924 \mathrm{~Hz})$ cancellation. As shown, the tone is cancelled by $5 \mathrm{~dB}$, which corresponds to a noticeably quieter tone, subjectively speaking. The reason that the reduction at the $8^{\text {th }}$ order is larger than the $24^{\text {th }}$ is due the fact that the $24^{\text {th }}$ order is much quieter, so it does not require as much cancellation as the $8^{\text {th }}$ order.

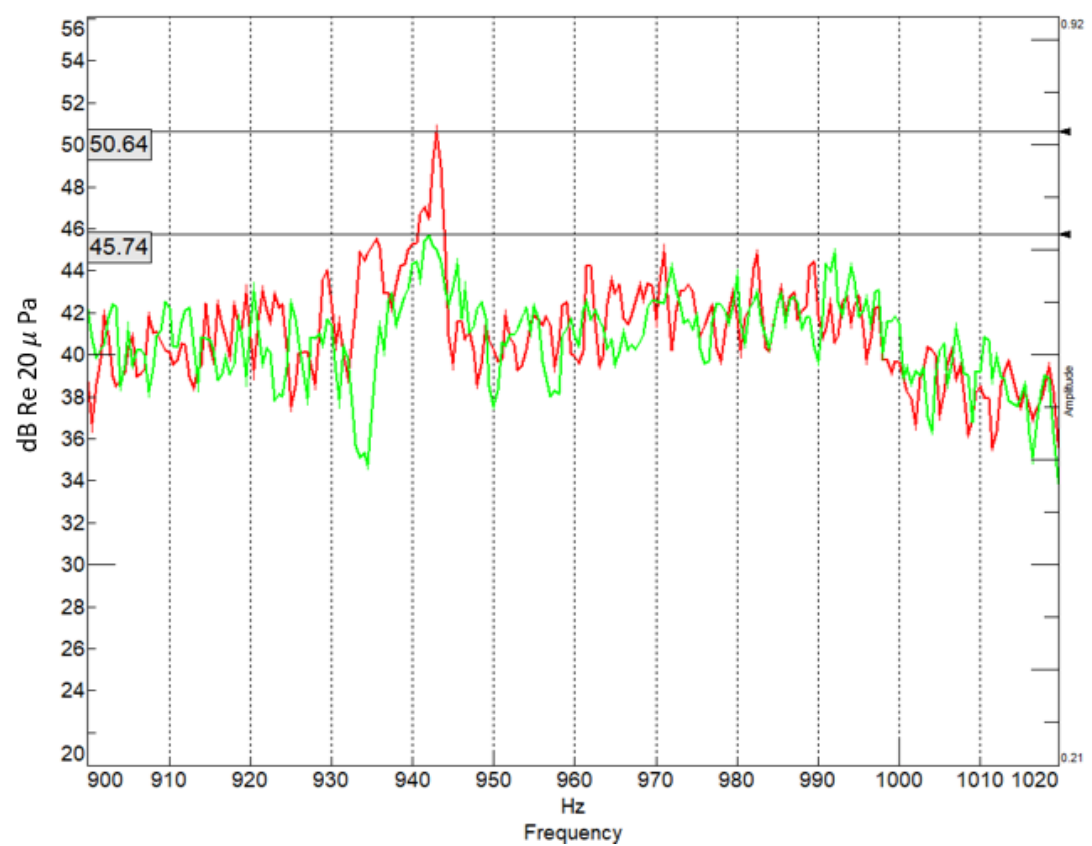

Fig. 30 A $24^{\text {th }}$ order cancellation test. Red data is cancellation off, and green data is cancellation on. The frequency of interest was $942 \mathrm{~Hz}$.

The next portion tested was to see if the system could handle more than 1 order at a time and successfully cancel with the CNT speaker. The orders tested this time were $8^{\text {th }}$ order $(314 \mathrm{~Hz})$ and the $16^{\text {th }}$ order $(627 \mathrm{~Hz})$. During test it was found that both the $8^{\text {th }}$ order and $16^{\text {th }}$ order tests were consistently able to cancel. After 5 repeat tests, the reduction for the $8^{\text {th }}$ order had a mean value of $18.6 \mathrm{~dB}$ and the $16^{\text {th }}$ order had a mean value of $9 \mathrm{~dB}$. Not only was the ANC system able to consistently converge on the wave to cause cancellation, but an additional 3-minute test showed that the system could consistently cancel the tones. Figure 31 shows the 2-order cancellation and figure 32 shows the 2-order cancellation over a stable period of 3 minutes. The reason 3 minutes was chosen was for its short-term observation of cancellation. Long-term cancellation has yet to be tested, but it is predicted that there should not be any long-term cancellation issues with this system as long as the tachometer is functioning properly. 




Fig. $318^{\text {th }}(314 \mathrm{~Hz})$ and $16^{\text {th }}(627 \mathrm{~Hz})$ orders cancellation data. Here the red shows the cancellation off and the cyan shows the cancellation on.

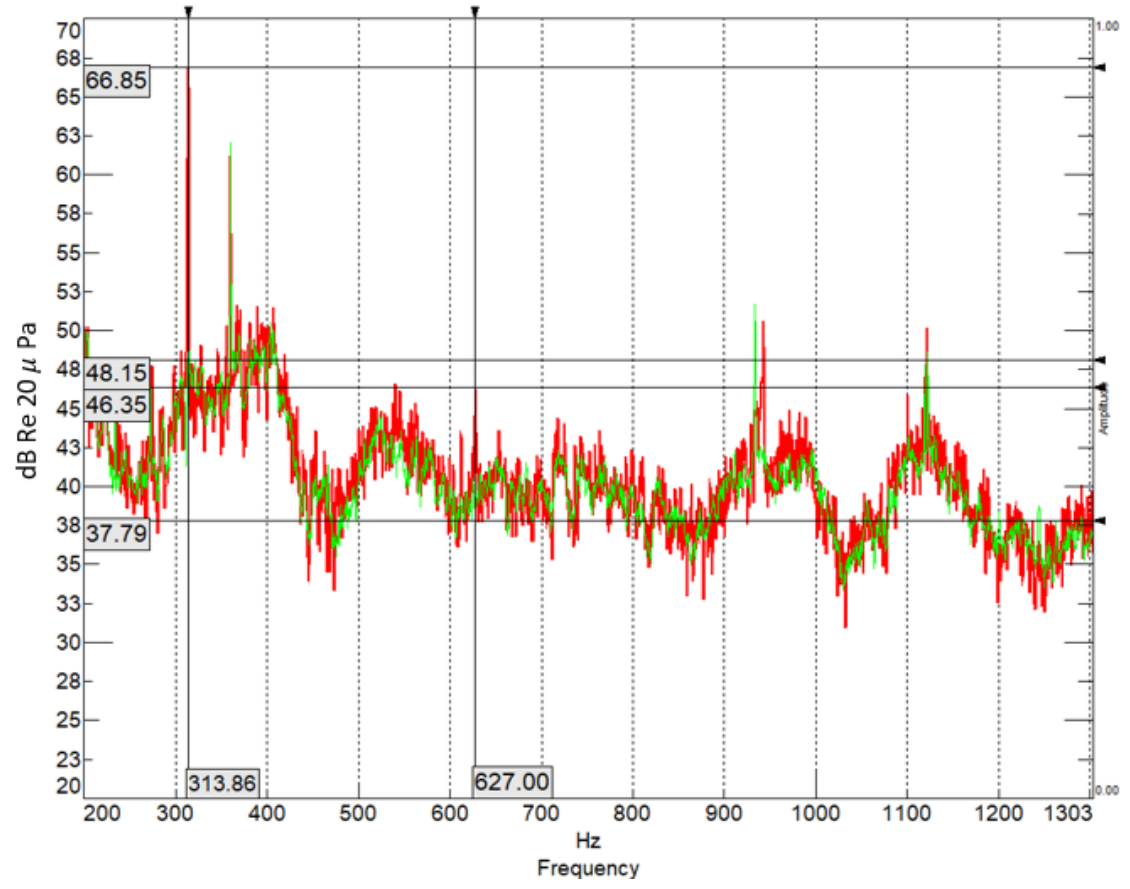

Fig. 32 A three-minute stability test for the cancellation of $8^{\text {th }}$ and $16^{\text {th }}$ orders. The red shows the cancellation off and the green shows the cancellation on. 


\subsection{Non-Planar Wave Cancellation}

When Cancelling non-planar waves, the two methods used were off center excitation and centered excitation. This was to excite at least two different types of modes, the "donut mode" $(1,0)$ mode and the circumferential mode $(0,1)$.

\subsubsection{Centered in the pipe}

To test the cutoff frequency limits of the ANC system, a $1250 \mathrm{~Hz}$ tone was played through the speaker. This tone is right below the calculated cut-on frequency of $1318 \mathrm{~Hz}$, so it was predicted to be successful at cancelling this tone. Figure 33 shows the cancellation test for the $1250 \mathrm{~Hz}$ tone.

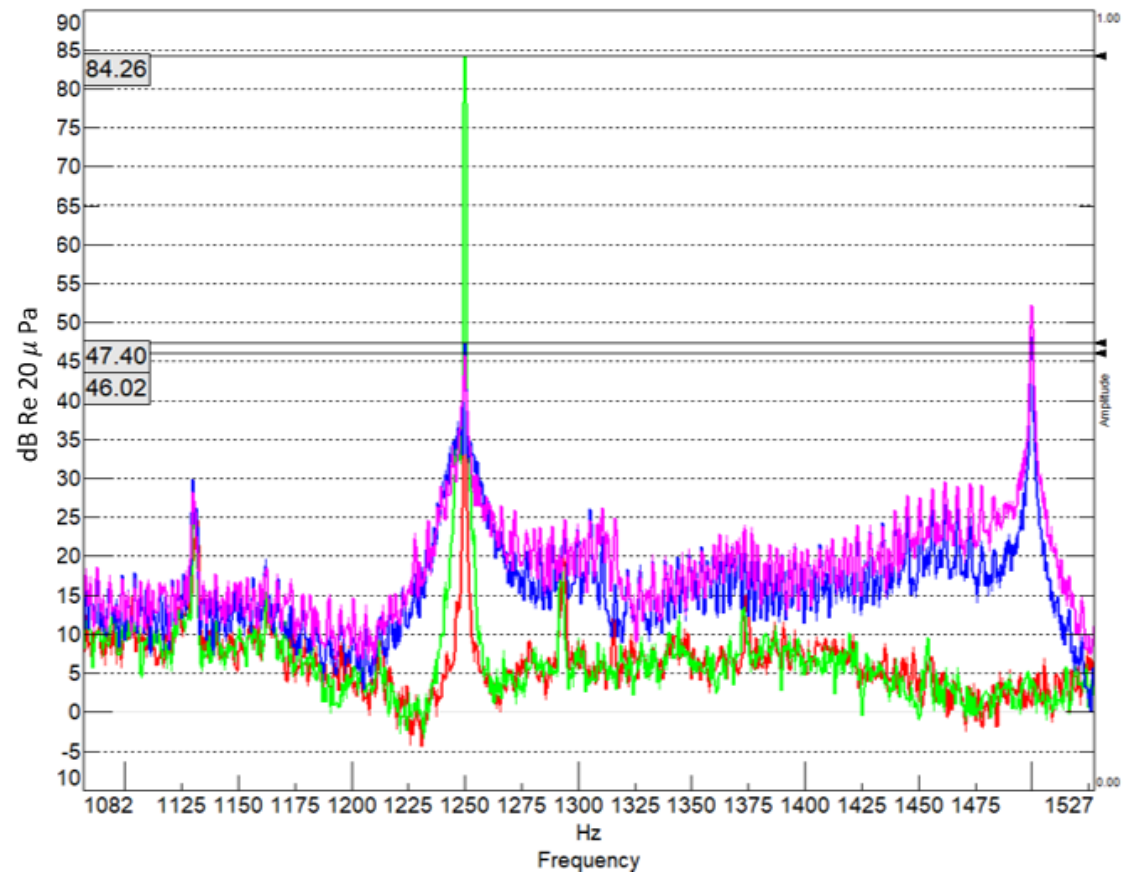

Fig. 33 Cancellation of a $1250 \mathrm{~Hz}$ tone that is before the cutoff frequency. The red and green data are cancellation off and the blue and magenta are two cancellation on runs.

As shown above, the two tests show a cancellation of around $37 \mathrm{~dB}$. This is positive and initially unexpected for this system since it was theorized to potentially have some issues with cancelling at higher frequencies. The two main issues suspected were the tracking algorithm having a difficult time tracking higher frequencies, and the system characterization that is more sensitive to errors in the phase approximation. With this result, the cut-on frequency was tested. Figure 34 shows the results of testing at the cut-on frequency. Here, it was seen that the cancellation was roughly $45 \mathrm{~dB}$. This appears to be positive on the ability for the system to cancel at the cut-on frequency while the disturbance is centered in the duct. Testing above the cut-on frequency, the frequency of $1400 \mathrm{~Hz}$ was chosen, as this would ensure that the duct is the in nonplanar range. 
Seen in figure 35, when the disturbance is centered in the duct, a cancellation of 47 $\mathrm{dB}$ can be achieved. This can mean that the speaker can cancel axially symmetric modes due to the geometry of the speaker being axially symmetric. These preliminary results show that the ANC system may be affective at cancelling for this type of application.



Fig. 34 Cut on Frequency cancellation test. Here, the red and green show cancellation is off, and the magenta and cyan show cancellation is on at the $1318 \mathrm{~Hz}$ tone. 


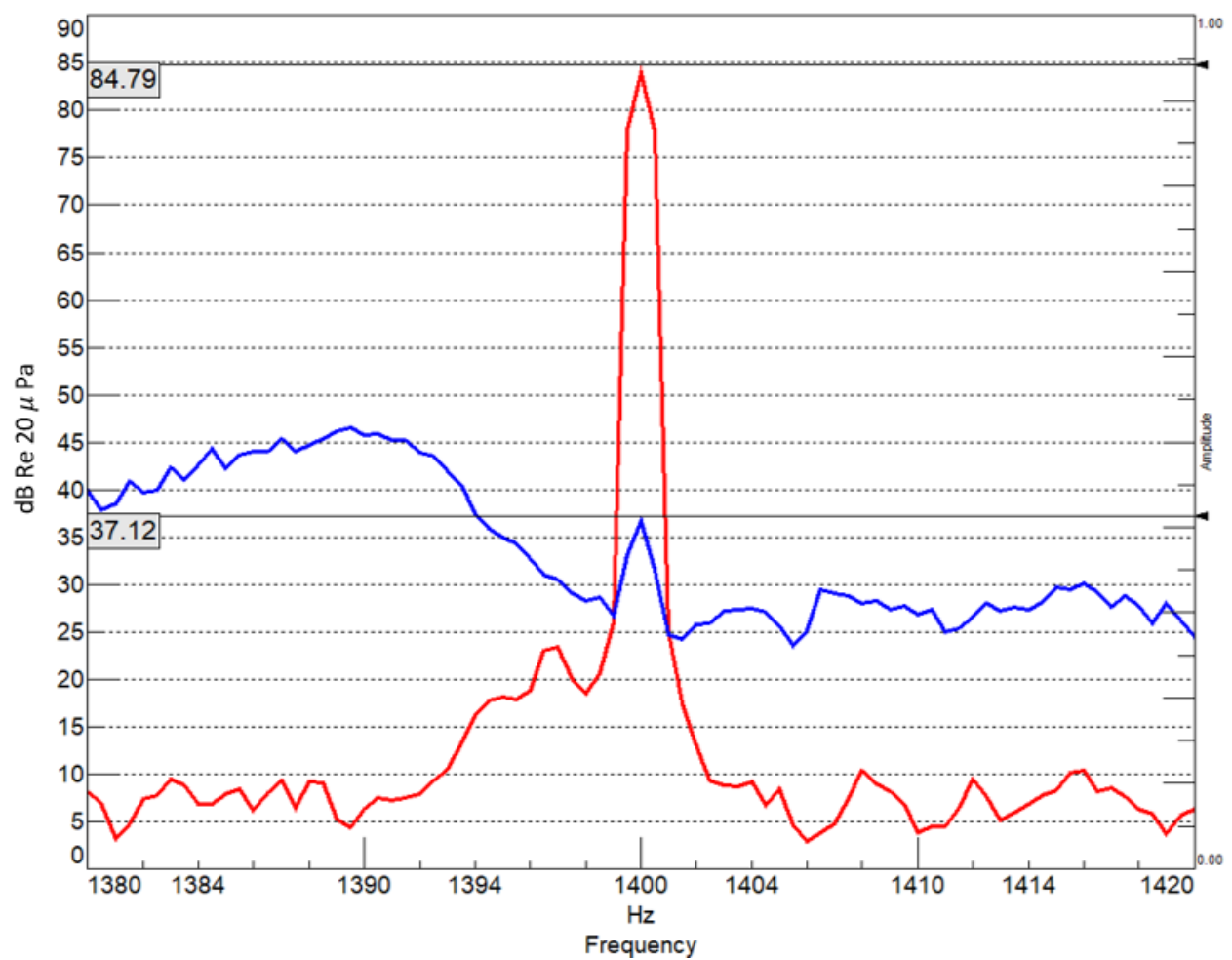

Fig. 35 Cancellation above the cut-on frequency at $1400 \mathrm{~Hz}$. The red data shows cancellation off and blue data shows cancellation on.

One final test was conducted above the cut-on frequency at $1500 \mathrm{~Hz}$. This was to once again test the non-planar contribution versus the planar contribution of the disturbance. Seen in figure 36, the cancellation still yields $35 \mathrm{~dB}$ in tonal reduction, but the algorithm begins to have a difficult time cancelling, and outputs other frequencies around the $1500 \mathrm{~Hz}$ tone. Although the overall sound level is reduced, the ANC system does have difficulty only outputting the single tone. More testing into this phenomenon needs to be conducted. 


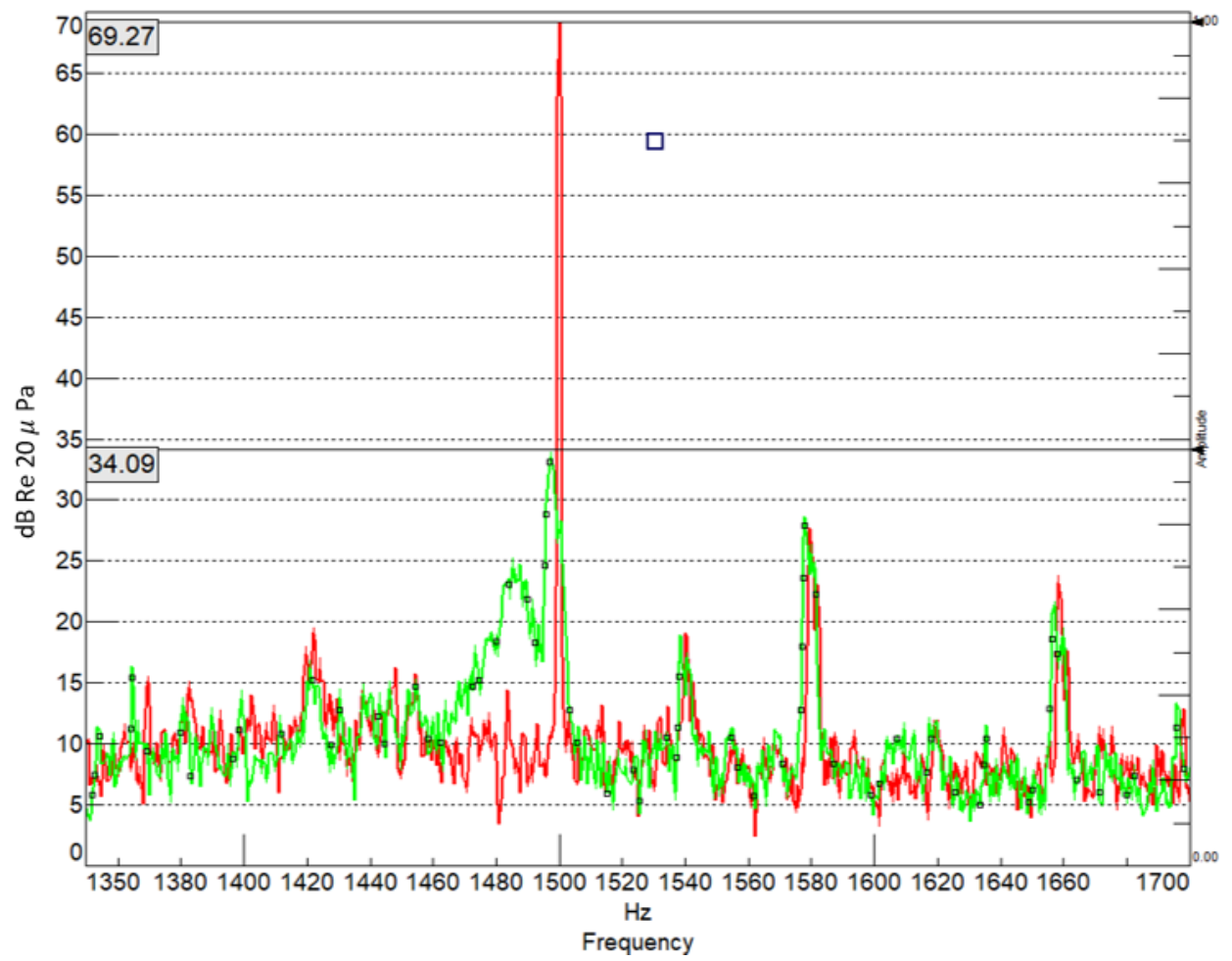

Fig. 36 Cancellation at $1500 \mathrm{~Hz}$. The red shows cancelation off, and the green data shows cancellation on.

\subsubsection{Off Centered in Pipe}

To excite modes that are not a "donut" shape, off centered testing was done in the pipe. Once again, a tone was chosen that was before the cut-on frequency. Here this tone was $1200 \mathrm{~Hz} .1200 \mathrm{~Hz}$ was used instead of 1250 because during characterization, there was a dip in the coherence at $1250 \mathrm{~Hz}$. Figures 37 and 38 show the $1200 \mathrm{~Hz}$ cancellation test. The octave map was chosen to highlight how the ANC algorithm was malfunctioning and outputting sound pressure at other frequencies in the pipe. In figure 37 , the original tests show that the ANC system was able to cancel $17 \mathrm{~dB}$ and $40 \mathrm{~dB}$ for the two runs respectively. The system does, however, output other tones that cause the total sound level to go up. While the total sound level for this test was still lower with the active system than without, this was not the case for later testing at the cut-on frequency. 


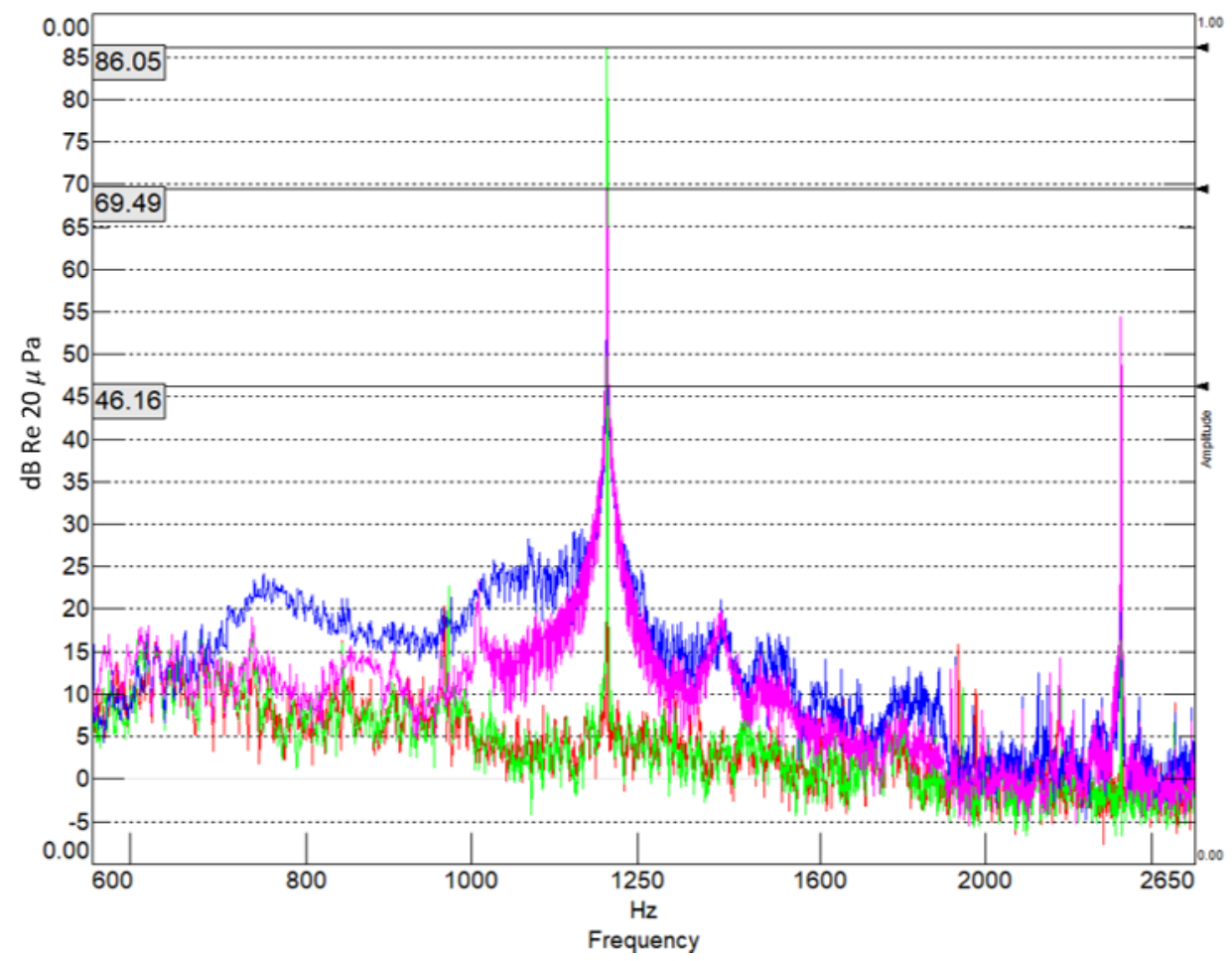

Fig. 37 Off centered cancellation of the $1200 \mathrm{~Hz}$ tone. Red and green are the cancellation off runs and the blue and magenta are cancellation on runs.

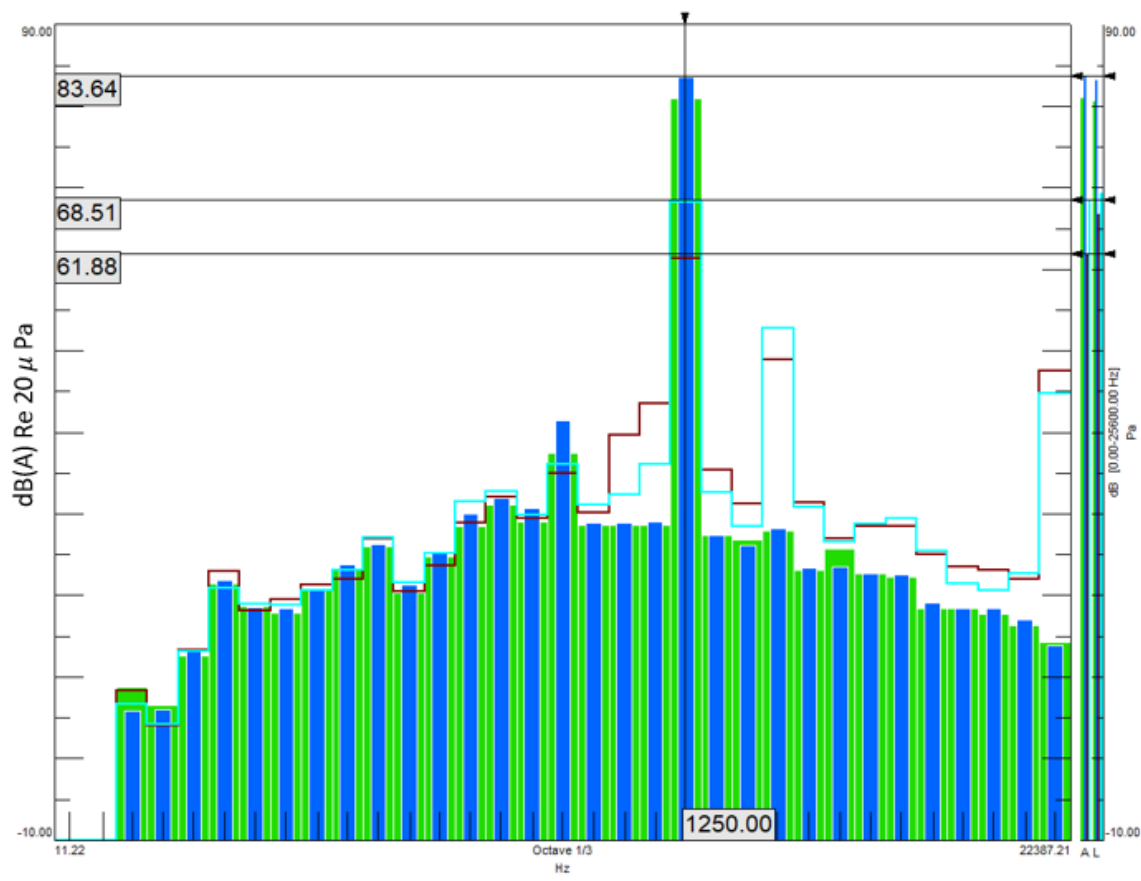

Fig. 38 1/3 Octave map of the two cancellation tests. The blue and green bars show cancellation off and the cyan and brown bars show cancellation on. 
A test at the cut-on frequency was conducted with the disturbance off centered in the pipe. Figures 39 and 40 show the frequency spectra and octave data for this test. At the cut-on frequency, there is some cancellation experienced, approximately $5 \mathrm{~dB}$, however there is also other tones that are being generated that cause peaks in the sound data. This appears to be an algorithm problem. The ANC was outputting the correct $1318 \mathrm{~Hz}$ tone but was also outputting harmonics of a $30 \mathrm{~Hz}$ tone (see figure 39). The result is the total sound level for cancellation on was higher than it was when off. The cause of this is theorized to be electrical interference from the signal generator used to produce the pure tones for this testing. Further ANC testing needs to be compiled to extract the root cause and prevent interference in the drive signal production.

The next tone tested was the $1400 \mathrm{~Hz}$ tone, above the cut-on frequency. Here it is shown in figures 41 and 42 that cancellation was not achieved at this frequency. Instead, the ANC system contributed to a higher noise level by playing $30 \mathrm{~Hz}$ harmonics again. Since no cancellation occurred, it is theorized that the ANC algorithm could not lock on the tachometer signal properly or that it failed to optimize during FXLMS algorithm. The total sound output was increased by $12 \mathrm{~dB}$ after cancellation was turned on. These are the worst results recovered from testing. Typically, even above the cut-on frequency, some portion of the wave was cancelled and minimal cancellation occurred. However, it appears there is an issue with the ANC system that is unaccounted for.

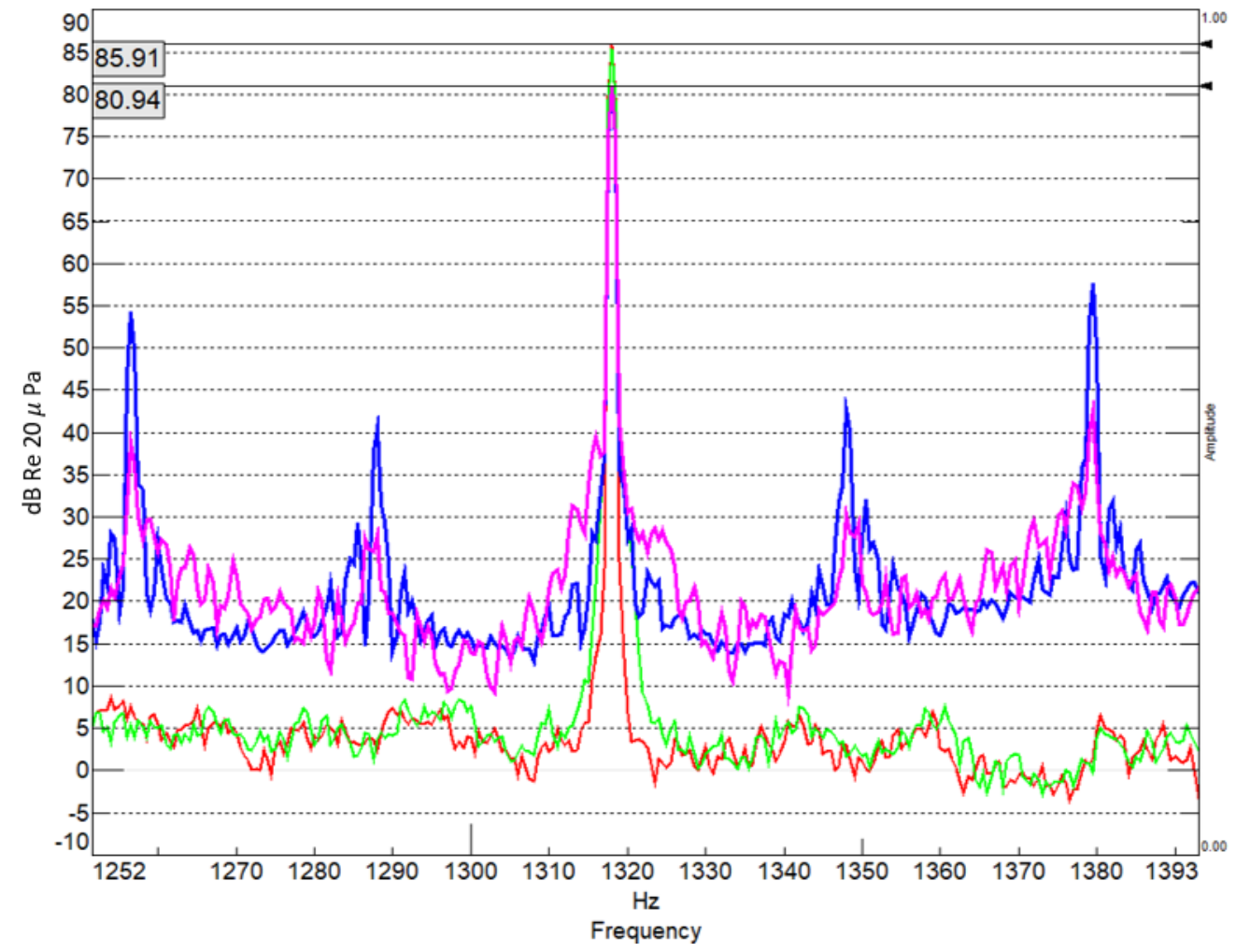

Fig. 39 Cancellation at $1318 \mathrm{~Hz}$ with disturbance off centered. Red and green show the cancellation off and blue and magenta show cancellation on. 


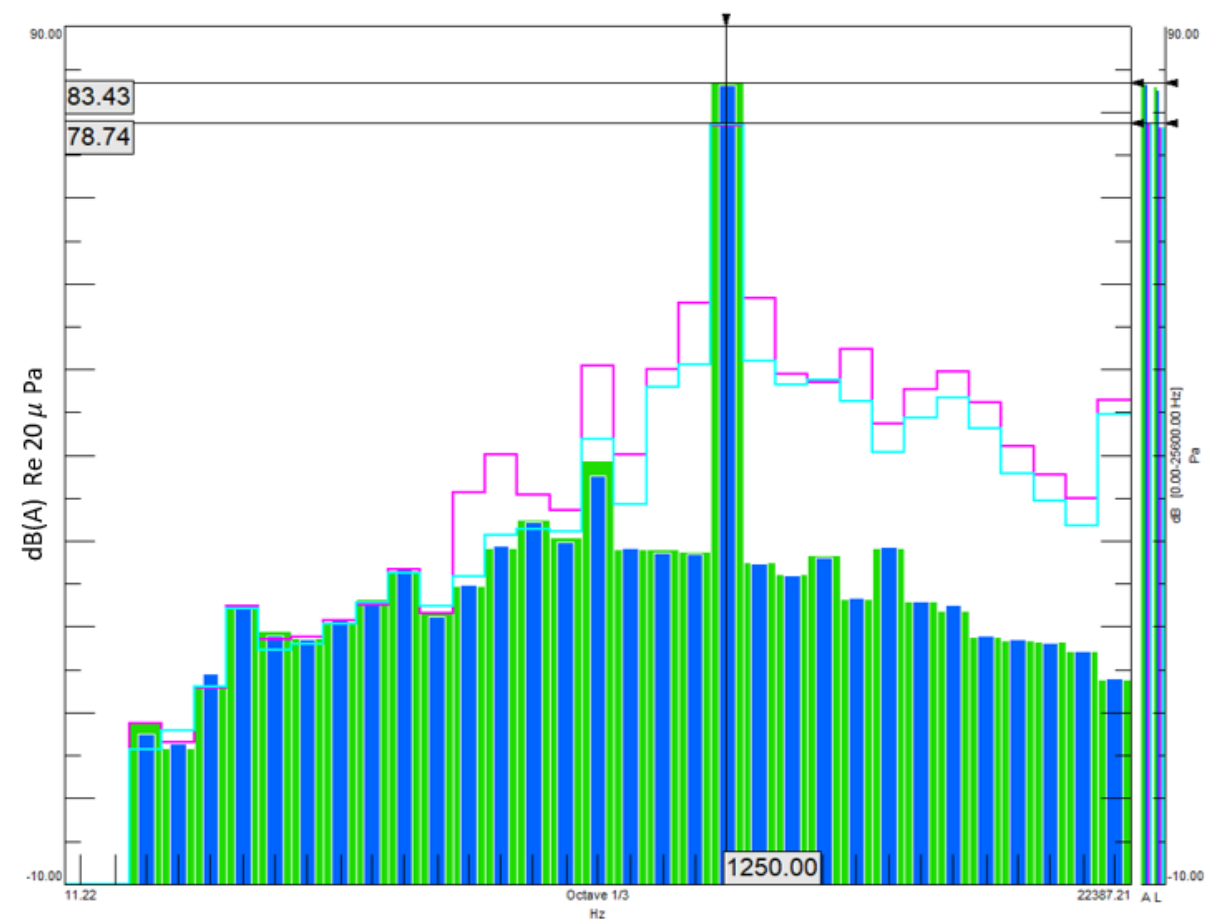

Fig. 40 1/3 Octave band spectra of cancellation at $1318 \mathrm{~Hz}$ with disturbance off centered. Blue and green show the cancellation off and cyan and magenta show cancellation on.

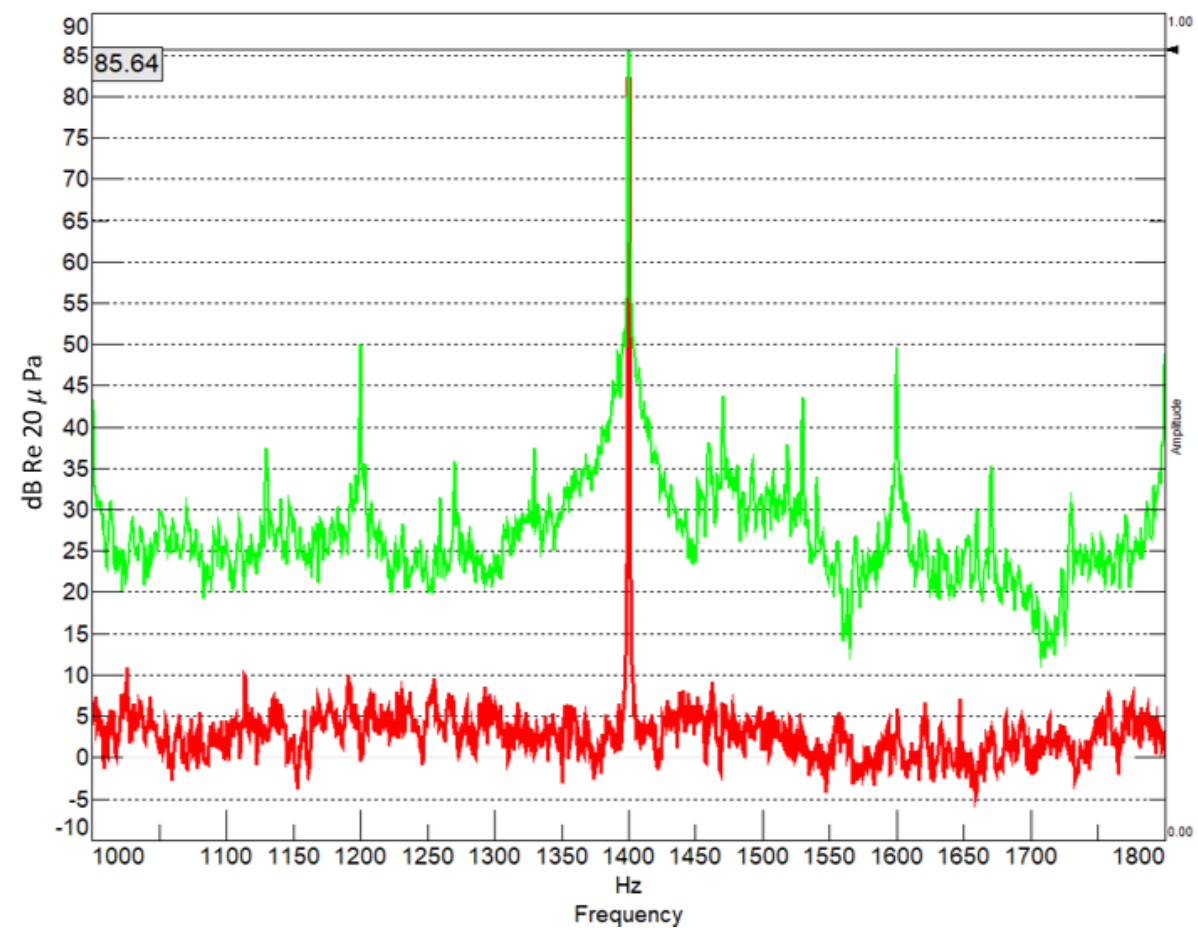

Fig. 41 Cancellation at $1400 \mathrm{~Hz}$ with disturbance off centered. Red shows the cancellation off and green shows cancellation on. 


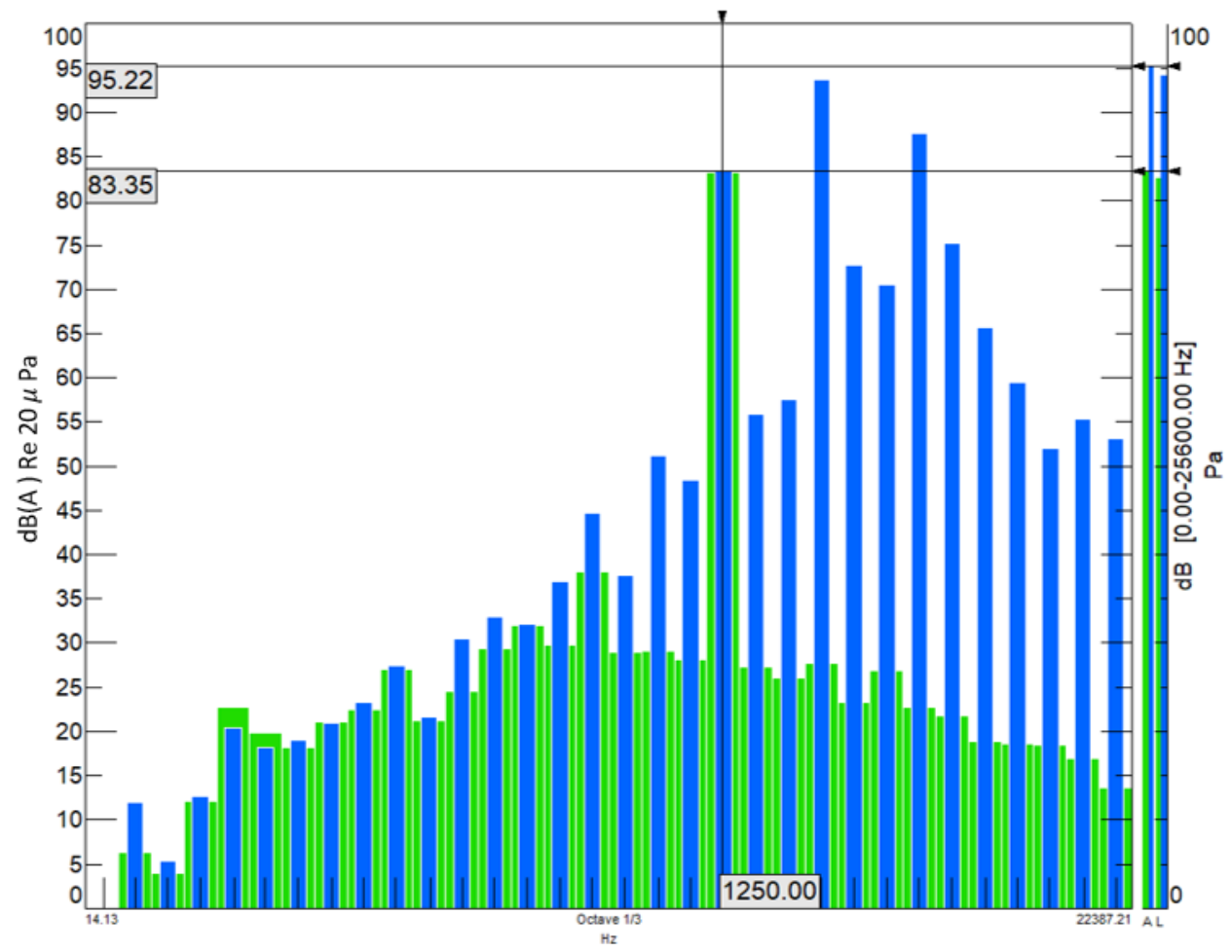

Fig. 42 1/3 Octave band spectra of cancellation at $1400 \mathrm{~Hz}$ with disturbance off centered. Green shows the cancellation off and blue shows cancellation on.

Downstream sound pressure was measured to determine if the sound cancellation done during non-planar testing was local or global. To examine this, a microphone and wind screen was placed 3.5 feet, or 7 pipe diameters away from the duct. The $1318 \mathrm{~Hz}$ cuton frequency test was performed, and it was found that local cancellation was mostly occurring at this frequency. Locally, the system was cancelling $40 \mathrm{~dB}$ of sound at the target frequency. Globally, however, the system was only cancelling $5 \mathrm{~dB}$. While this cancellation system is still a positive, it means the algorithm needs to be improved so that the receiver sees the $40 \mathrm{~dB}$ of cancellation. Subjectively, during testing, it was noted that the sound was noticeably quieter. This observation is consistent with the downstream pipe measurement data. Figures 43, 44, and 45 show the downstream testing data of $1318 \mathrm{~Hz}$, $1400 \mathrm{~Hz}$, and $1500 \mathrm{~Hz}$ respectively. All data show that global cancellation was occurring, but only about $5 \mathrm{~dB}$ consistently. 


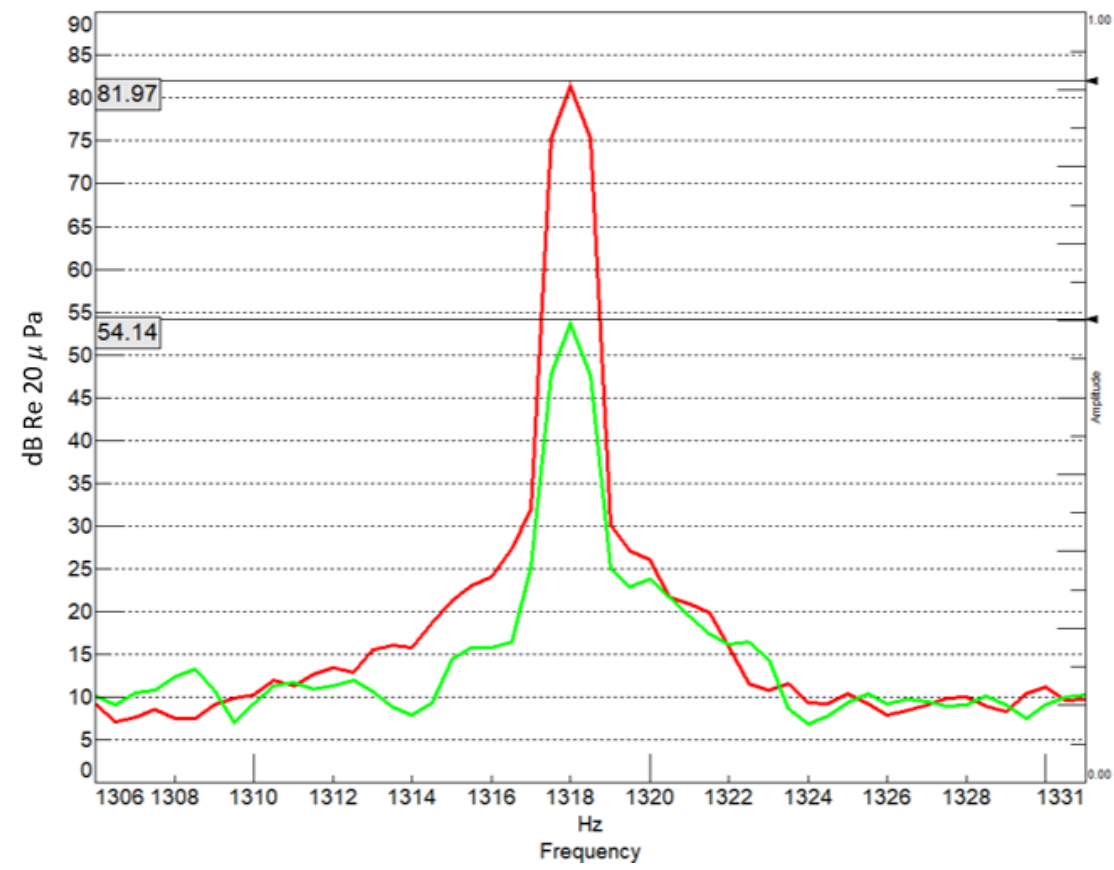

Fig. 43 Downstream testing of $1318 \mathrm{~Hz}$ tone. The red indicated the near duct microphone measurement with cancellation off. The green shows the near duct microphone with cancellation on. Blue shows the downstream microphone with cancellation off and magenta shows the downstream microphone with cancellation on.

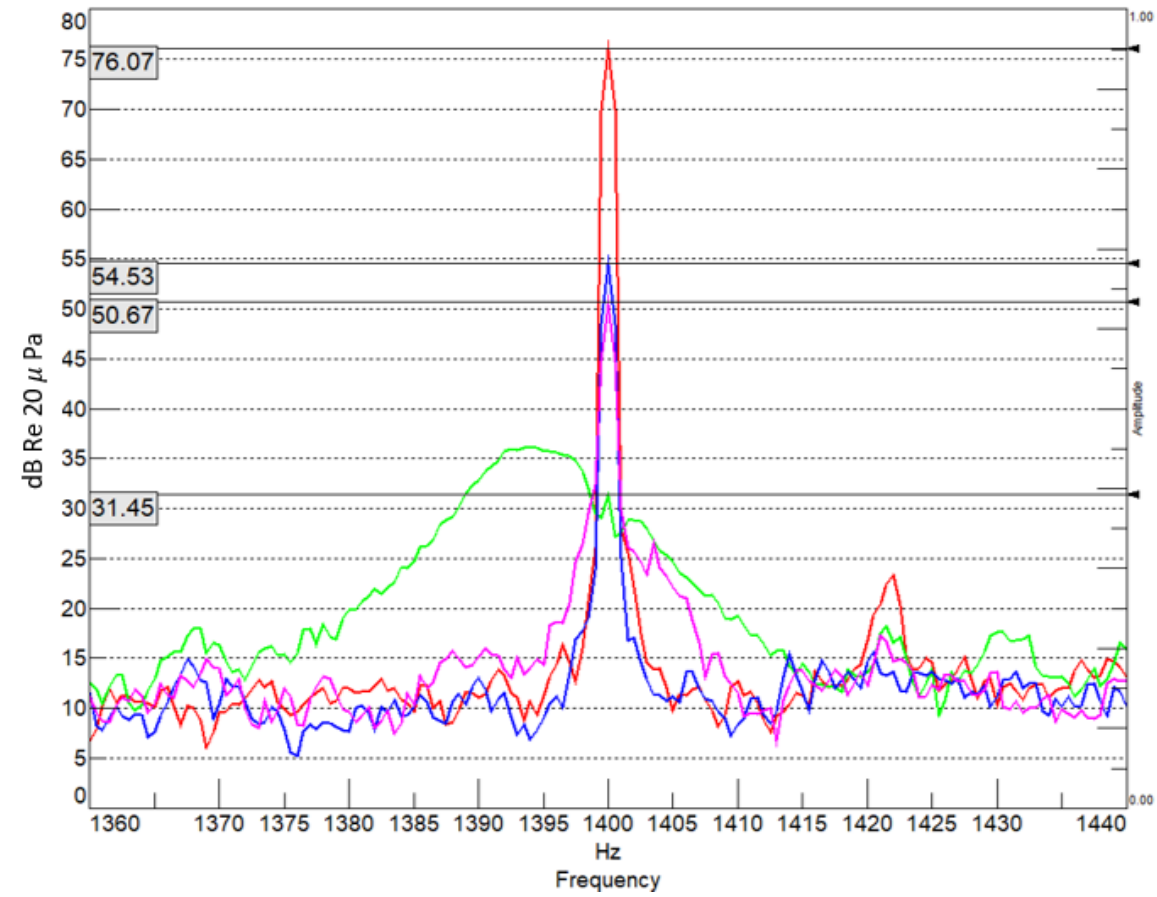

Fig. 44 Downstream testing of $1400 \mathrm{~Hz}$ tone. The red indicated the near duct microphone measurement with cancellation off. The green shows the near duct microphone with 
cancellation on. Blue shows the downstream microphone with cancellation off and magenta shows the downstream microphone with cancellation on.

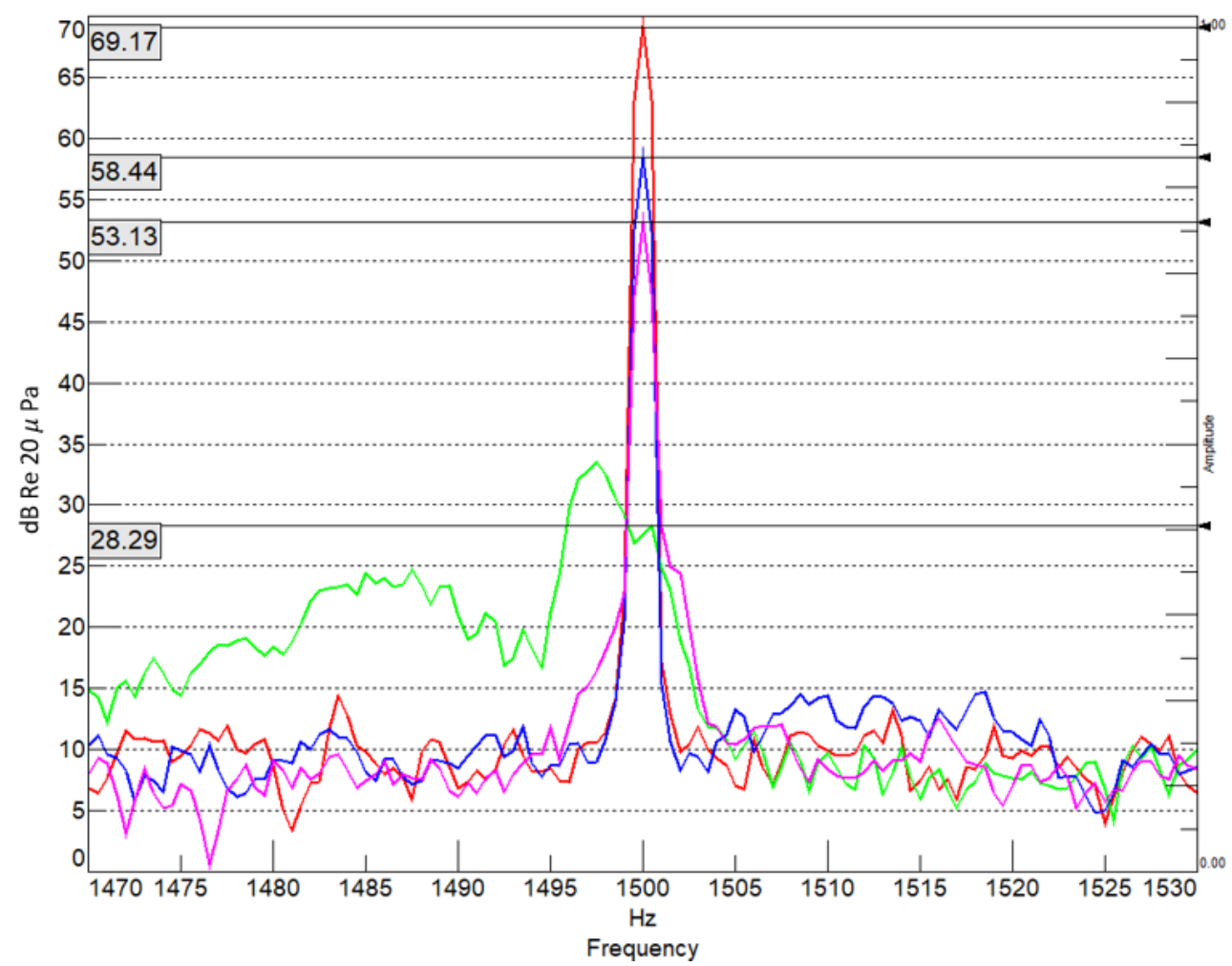

Fig. 45 Downstream testing of $1500 \mathrm{~Hz}$ tone. The red indicated the near duct microphone measurement with cancellation off. The green shows the near duct microphone with cancellation on. Blue shows the downstream microphone with cancellation off and magenta shows the downstream microphone with cancellation on. 


\subsection{Accelerated Life Testing}

During accelerated life testing, two different tests were conducted, over the course of 3 hours. The initial power data for the first test became corrupted, so an additional 3hour test was conducted. The test was conducted by heat cycling the CNT at a rate of 20 $\mathrm{KHz}$. This is 63 times more than the expected run rate of $314 \mathrm{~Hz}$ during use. At the target usage of $314 \mathrm{~Hz}$, the CNT was life tested for an equivalent of 382.2 hours. During the first test, sound data was still recorded and showed similar steady results to the second sound test. The data could not be processed the same, but it can be assumed that the initial 3-hour test had steady sound and voltage readings as well since the same waveform and settings were used. The second test produced steady power, voltage, and current results across the 3-hours. These are successful results as changes in resistance and power indicate that sections of the CNT have become damaged. The consistent power measurements show that the CNT was function as intended and did not burn up during testing. The voltage averaged at $27.6 \mathrm{~V}$, the current was 2.59 Amps, and the power was 71.6 Watts. Figure 46 shows the electrical traces over time for the speaker as well as sound pressure at $20 \mathrm{KHz}$ over time.
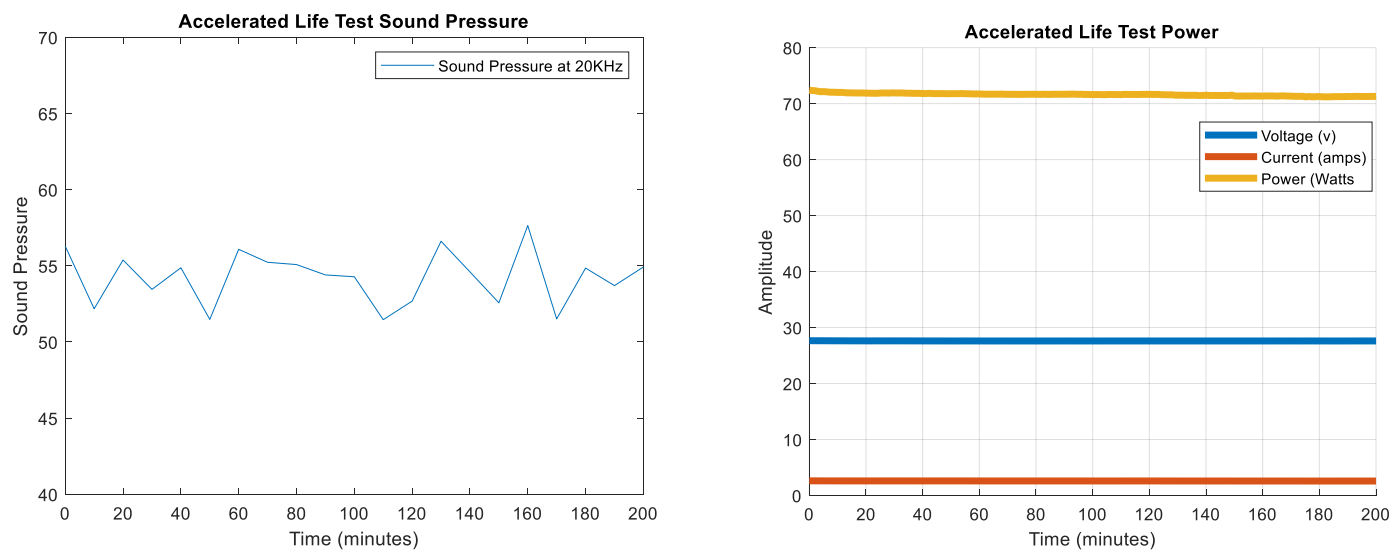

Fig. 46 Sound pressure at $20 \mathrm{KHz}$ recorded at 10 minute increments (left) and voltage and current measurements taken at 1 minute increments (right).

After inspection, the speaker appeared to be undamaged. A manual temperature test of the duct during testing showed that with the fan running, the CNT did not heat the duct beyond room temperature. This is a contrast to non-planar testing where no fan was present. Here, the CNT speaker overheated the duct and burnt through the CNT backing materialbreaking the speaker. During this fail condition, the resistance of the speaker slowly increased until it reached $70 \Omega$ and the amount of energy that the preamp could produce was not enough to power the speaker. The failed speaker can be found in fig. 47. The new CNT speaker that was cooled by the fan did not exhibit any fail conditions even after heat cycling for 6 hours. This provides evidence that a HVAC cooling system is a possible solution for CNT thermal management. It may also provide make the speaker more efficient since CNT speakers work on a thermal gradient-meaning that the colder it is, the louder the speaker can produce sound. 


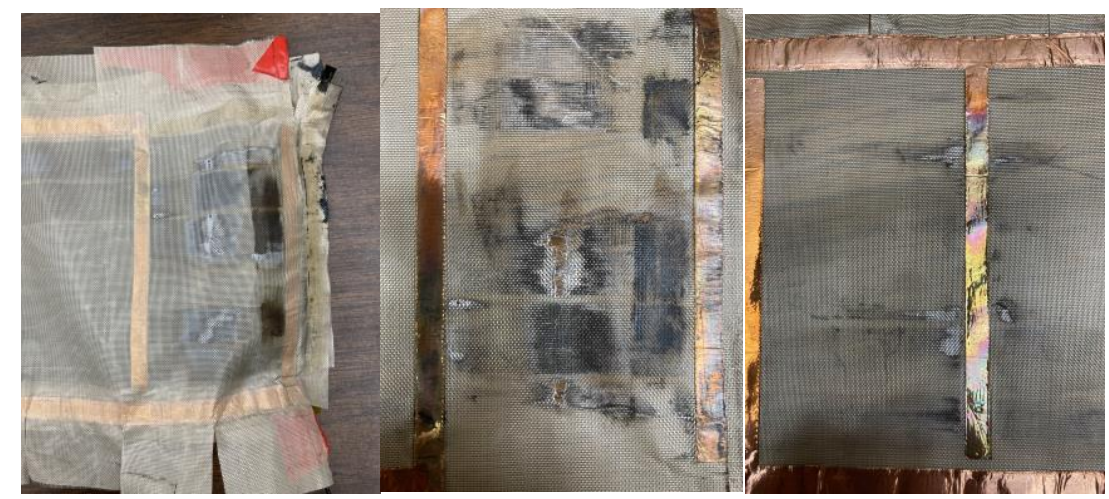

Fig. 47 Various images of a failed CNT speaker that burned out due to lack of cooling. 


\section{Conclusions}

\subsubsection{Speaker Construction}

Of all the speakers previously designed in this lab, this particular design shows the most promise. This design was easy to construct, and the electrode configuration created an optimal resistance for the speaker. With the discovery of the PTFE FG, the material allows the CNT to be protected and remain robust, while ensuring that the material itself is not damaged during use. The flexible material also allows the speaker to be rolled into the duct. The Kapton tape also allows the joints to be firmly connected while providing additional heat shielding. In previous designs, glue and tape used to construct the speaker has melted, causing failures. The new addition of Kapton allows the speaker to remain connected even after hours of use. Even after the speaker experienced general wear from moving and installing it, the layers of PTFE FG prevented damage. The other main benefit of the PTFE FG was that there was not a noticeable difference in sound output due to the holes in the woven cloth that allowed the sound to travel. Similarly, the weave size was small enough that dust did not get trapped; When this happens, the dust combusts and can cause cascade failures along the CNT, which increases the resistance. The only portions that need to be improved upon are removing human error factors, i.e. creating a cylinder that is computer operated and not human operated, and creating a better system for installing the speaker. Currently, the method used to quickly install the speaker was to use electrical tape and tape the speaker around the duct. This would not be easy to do if the speaker needed to be in the middle of the duct. Furthermore, some of the tape around the wires experienced melting and burning if the speaker was ran without the cooling system. Future iterations of installation need to be done for this speaker to be a commercially viable product. It is also shown in research that adding an air gap between the CNT and backing material can provide louder sound pressure [30] [31], so a future iteration may incorporate this into design for better efficiency.

\subsubsection{ANC Planar Cancellation}

As demonstrated in this paper, the CNT speaker coupled with the ANC system was able to successfully cancel tonal noise upon its first attempt. With the microphone located near the speaker, it was shown that for dominate tones, the speaker could reduce the sound levels up to $18.6 \mathrm{~dB}$. For non-dominant tones, the CNT speaker averaged around $9 \mathrm{~dB}$, which is still subjectively $2 \mathrm{x}$ as quiet as before the system was installed. The CNT speaker did demonstrate that it was capable of putting out acceptable sound pressure levels required to cancel the fan noise, without overstressing the speaker. The overall sound level was not noticeable, and with the broadband system in place, the actual tonal cancellation was also not noticeable. It is recommended that this system be used with either a broadband cancellation system, or a fan that is highly tonal. 


\subsubsection{ANC Non-planar cancellation}

The non-planar wave cancellation was considered a partial success. When the mode is axis-symmetric, the cancellation seen was around $40 \mathrm{~dB}$. The main drawbacks of nonplanar cancellation were that the ANC system seemed to have issues cancelling when the disturbance was off center of the pipe. Another key issue was that the system appeared to only cancel locally above the cut-on frequency. Global cancellation was approximately 5 $\mathrm{dB}$ and local cancellation was much higher. Global cancellation can be improved by accepting multiple microphone inputs into the ANC controller and allowing the algorithm to minimize the sound pressure seen across all of the microphone locations. The $5 \mathrm{~dB}$ sound reduction is still considered a success at these frequencies. In a real-life test setup, the system, in its current state, may output $30 \mathrm{~Hz}$ harmonics, but it is expected to still reduce the sound output overall. The $30 \mathrm{~Hz}$ harmonics would be masked in the flow noise of the fan. For disturbances that are centered in the pipe, the system performed well. Since most HVAC fans are centered within the duct, the application of this system to HVAC is expected to be good.

\subsubsection{Accelerated Life Testing}

The accelerated life test showed that the speaker could successfully heat cycle 20 $\mathrm{KHz}$ for at least 6 hours. 3 hours of which, it had a steady power output of $72 \mathrm{Watts}$ and a sound pressure of $56 \mathrm{~dB}$ at $20 \mathrm{KHz}$. This means that the CNT could theoretically run at the target frequency of $314 \mathrm{~Hz}$ for more than 63 hours straight. With this preliminary test data, it is thought that the CNT speaker could make an excellent solution for an ANC system. The long-term durability needs to be further tested at different fan speeds and different power levels. Future testing needs to be done on CNT power density versus fan output. Different cooling rates may have an impact on CNT; however, only one fan speed was tested. 


\section{Reference List}

[1] A. E. Aliev, "Thermophones using carbon nanotubes and alternative nanostructures for high power sound generation and noise cancellation," Inter Noise, 2014.

[2] W. Xiao-Di, K. Vinodgopal and D. Gui Ping, "Synthesis of Carbon Nanotubes by Catalytic Chemical Vapor Deposition, Perspective of Carbon Nanotubes," Intechopen, 2018. [Online]. Available: https://www.intechopen.com/books/perspective-of-carbon-nanotubes/synthesis-ofcarbon-nanotubes-by-catalytic-chemical-vapor-deposition.

[3] K. K. Lau, J. Bico, K. B. Teo, M. Chhowalla, G. A. J. Amaratunga, I. W. Milne, G. H. Mckinley and K. K. Gleason, "Superhydrophonic Carbon Nanotube Forests," Nano letters, vol. 3, no. 12, pp. 1701-1705, 2003.

[4] X. Yu, R. Rajamani, K. Stelson and T. Cui, "Carbon nanotube-based transparent thin film acoustic actuators and sensors.," Sensors and Actuators A: Physical, pp. 626-31, 2006.

[5] K. Suzuki, S. Sakakibara, M. Okada, Y. Neo, Mimura H, Y. Inoue and et al., "Study of carbon-nanotube web thermoacoustic loud speakers," Japanese Journal of Applied Physics, 2011.

[6] S. Xie, W. Li, Z. Pan, B. Chang and L. Sun, "Mechanical and physical properties on carbon nanotube," Journal of Physics and Chemistry of Solids, vol. 61, no. 7, pp. 1153-1158, 2000.

[7] S. Hu, Carbon nanotube thin films for active noise cancellation, solar energy harvesting, and energy storage in building windows., Minneapolis, MN: University of Minnesota, 2014.

[8] T. M. Bouman, A. R. Barnard and M. Asgarisabet, "Experimental quantifications of the true efficiency of carbon nanotube thin-film thermophones," The Journal of the Acoustical Society of America, vol. 139, no. 3, pp. 1353-1363, 2016.

[9] A. Barnard, D. Jenkins, T. Brungart, T. McDevitt and B. Kline, "Feasibility of a high-powered carbon nanotube thin-film loudspeaker," The Journal of Acoustical Society of America, pp. EL276-EL81, 2013.

[10] H. Arnold and I. Crandall, "The thermophone as a precision source of sound," Physical Review, pp. 10-22, 1917. 
[11] L. Xiao, Z. Chen, C. Feng, L. Liu, Z.-Q. Bai, Y. Wang and et al. , "Flexible, stretchable, transparent carbon nanotube thin film loudspeakers," Nano letters, pp. 4539-45, 2008.

[12] X. Xiao, P. Liu , Q. Li, Z. Feng, S. Fan and et al., "High frequency response of carbon nanotube thin film speakers in gases," Journal of Applied Physics, 2011.

[13] C. Lim, L. Tong and Y. Li, "Theory of suspended carbon nanotube thinfilm as a thermal-acoustic source.," Journal of Sound and Vibration, pp. 5451-61, 2013.

[14] M. Asgarisabet, A. R. Barnard and T. M. Bouman, "Near field acoustic holography measurements of carbon nanotube thin film speakers," Journal of the Acoustical Society of America, vol. 140, no. 6, pp. 4237-4245, 2016.

[15] A. E. Aliev, N. K. Mayo, R. H. Baughman, D. Aviroyik, S. Priya, M. R. Zarnetske and J. B. Blottman, "Thermal management of thermoacoustic sound projectors using a free-standing carbon nanotube aerogel sheet as a heat source," Nanotechnology, vol. 25, no. 40, p. 405704, 2014.

[16] A. E. Aliev, Y. N. Garstein and R. H. Baughman, "Increasing the efficiency of thermoacoustic carbon nanotube sound projectors," Nanotechnology, vol. 24, no. 23, p. 235501, 2013.

[17] A. Barnard, T. Brungart, T. McDevitt, A. Aliev, D. Jenkins , B. Kline and et al, "Advancements towardsa high-power, carbon nanotube, thin-film loudspeaker," Noise Control Engineering Journal, vol. 62, pp. 360-7, 2014.

[18] V. Vesterinen, A. Niskanen, J. Hassel and P. Helisto, "Fundamental efficiency of nanothermophones: modeling and experiments," Nano letters, pp. 5020-4, 2010.

[19] S. Sidhu, Implementation of active noise cancellation in a duct, Barnaby, B.C., Canada: Simon Fraser University, 2013.

[20] H. Zhu, R. Rajamani, J. Dudney and K. Stelson, "Active noise controlusing a distributed mode flat panel loudspeaker," ISA transactions, vol. 42, pp. 475-84, 2003.

[21] S. Hu, R. Rajamani and X. Yu, "Active noise control for selective cancellatin of external disturbances," Proceesings od the 2011 American Control Conference, 2011.

[22] S. M. Kuo and D. R. Morgan, Active Noise Control Systems Algorithms and DSP Implementations, New York: John Wiley \& Sons, Inc. , 1996. 
[23] J. Carmona and V. Alvarado, "Active noise control of a duct using robust control theory," Proceedings of the 37th IEEEConference on Decision and Control, no. 98, p. C36171.

[24] J. Tichy, G. E. Warnaka and L. A. Poole, "A Study of Active Control of Noise in Ducts," Journal of Vibration and Acoustics, vol. 106, no. 3, pp. 399-404, Jan. 1984.

[25] M. L. Munjal, Acoustics of Ducts and Mufflers with Application to Exhaust And Ventilation System Design, New York: John Wiley \& Sons, Inc. , 1987.

[26] L. E. Kinsler, A. R. Frey, A. B. Coppens and J. V. Sanders, Fundamentals of Acoustics Fourth Edition, New Jersey: John Wiley and Sons, Inc., 1999.

[27] E. W. Weisstein, "Bessel Function of the First Kind," MathWorld--A Wolfram Web Resource, 2020. [Online]. Available: https://mathworld.wolfram.com/BesselFunctionoftheFirstKind.html.

[28] D. T. Blackstock, Fundamentals of Physical Acoustics, New York: John Wiley \& Sons, Inc. , 2000.

[29] "Ripple Tank Simulator," falstad.com, [Online]. Available: https://www.falstad.com/ripple/.

[30] W. Nelson, "Active Noise Control using Carbon Nanotube Thermophones: Case Study for an Automotive HVAC Application," Michigan Technological University, Houghton, 2020.

[31] L. Tong, C. Lim and Y. Li, "Gas-filled encapsulated thermal-acoustic transducer," Journal of Vibration and Acoustics, p. 051033, 2013.

[32] L. Tong, C. Lim, S. Lai and Y. Li, "Gap seperation effect on thermoacoustic wave generation by heated suspended CNT nano-thinfilm," Applied Thermal Engineering, pp. 135-42, 2015.

[33] M. Kozlov, C. Haines, J. Oh, M. Lima and S. Fang, "Sound of Carbon Nanotube Assemblies," Journal of Applied Physics, pp. 106-124, 2009.

[34] T. Sugimoto and Y. Nakajima, "Acoustic characteristics of a flexible sound generator based on thermoacoustic effect," Proceedings of Meetings on Acoustics: Acoustical Society of America, p. 030004, 2013. 
[35] Y. Wei , X. Lin, K. Jiang, P. Liu, Q. Li and S. Fan, "Thermoacoustic chips with carbon nanotube thin yarn arrays," Nano letters, pp. 4795-801, 2013.

[36] A. Aliev, "Thermophones using carbon nanotubes and alternative nanostructures for high power sound generation and noise cancellation," INTER-NOISE and NOISECON Congress and Conference Proceedings: Institute of Noise Control Engineering, pp. 3938-47, 2014. 